Faculty of Science

Faculty Publications

"Certain Inequalities Pertaining to Some New Generalized Fractional Integral Operators"

Hari Mohan Srivastava, Artion Kashuri, Pshtiwan Othman Mohammed, and Kamsing Nonlaopon

2021

(C) 2021 Srivastava et al. This is an open access article distributed under the terms of the Creative Commons Attribution License. http://creativecommons.org/licenses/by/4.0

This article was originally published at:

https://doi.org/10.3390/fractalfract5040160

Citation for this paper:

Srivastava, H. M., Kashuri, A., Mohammed, P. O., \& Nonlaopon, K. (2021). Certain inequalities pertaining to some new generalized fractional integral operators. Fractal and Fractional, 5(4), 160-181. doi.org/10.3390/fractalfract5040160. 


\title{
Certain Inequalities Pertaining to Some New Generalized Fractional Integral Operators
}

\author{
Hari Mohan Srivastava ${ }^{1,2,3,4}\left(\mathbb{D}\right.$, Artion Kashuri ${ }^{5}\left(\mathbb{D}\right.$, Pshtiwan Othman Mohammed ${ }^{6, *(D)}$ \\ and Kamsing Nonlaopon $7, *$ (D) \\ 1 Department of Mathematics and Statistics, University of Victoria, Victoria, BC V8W 3R4, Canada; \\ harimsri@math.uvic.ca \\ 2 Department of Medical Research, China Medical University Hospital, China Medical University, \\ Taichung 40402, Taiwan \\ 3 Department of Mathematics and Informatics, Azerbaijan University, 71 Jeyhun Hajibeyli Street, \\ Baku AZ1007, Azerbaijan \\ 4 Section of Mathematics, International Telematic University Uninettuno, I-00186 Rome, Italy \\ 5 Department of Mathematics, Faculty of Technical Science, University "Ismail Qemali", 9400 Vlora, Albania; \\ artion.kashuri@univlora.edu.al \\ 6 Department of Mathematics, College of Education, University of Sulaimani, Sulaimani 46001, Iraq \\ 7 Department of Mathematics, Khon Kaen University, Khon Kaen 40002, Thailand \\ * Correspondence: pshtiwan.muhammad@univsul.edu.iq (P.O.M.); nkamsi@kku.ac.th (K.N.)
}

Citation: Srivastava, H.M.; Kashuri, A.; Mohammed, P.O.; Nonlaopon, K. Certain Inequalities Pertaining to Some New Generalized Fractional Integral Operators. Fractal Fract. 2021, 5, 160. https://doi.org/ 10.3390 / fractalfract5040160

Academic Editors: Agnieszka B. Malinowska and Ricardo Almeida

Received: 6 September 2021

Accepted: 4 October 2021

Published: 9 October 2021

Publisher's Note: MDPI stays neutral with regard to jurisdictional claims in published maps and institutional affiliations.

Copyright: (c) 2021 by the authors. Licensee MDPI, Basel, Switzerland. This article is an open access article distributed under the terms and conditions of the Creative Commons Attribution (CC BY) license (https:// creativecommons.org/licenses/by/ $4.0 /)$.

\begin{abstract}
In this paper, we introduce the generalized left-side and right-side fractional integral operators with a certain modified ML kernel. We investigate the Chebyshev inequality via this general family of fractional integral operators. Moreover, we derive new results of this type of inequalities for finite products of functions. In addition, we establish an estimate for the Chebyshev functional by using the new fractional integral operators. From our above-mentioned results, we find similar inequalities for some specialized fractional integrals keeping some of the earlier results in view. Furthermore, two important results and some interesting consequences for convex functions in the framework of the defined class of generalized fractional integral operators are established. Finally, two basic examples demonstrated the significance of our results.
\end{abstract}

Keywords: Chebyshev inequality; generalized fractional integral operators; modified ML kernel; FW function; general Wright function and its companions and extensions; synchronous functions; integral inequalities

\section{Introduction}

Fractional calculus is the study of integrals and derivatives of arbitrary order which was a natural outgrowth of conventional definitions of calculus integral and derivative. There are several problems in the mathematics and its related real world applications wherein fractional derivatives occupy an important place, see [1-5]. Each conventional fractional operator with its own special kernel can be used in a certain problem. Analyzing the uniqueness of fractional ordinary and partial differential equations can be performed by employing fractional integral inequalities. In the literature many applications can be found, see [6-8].

The integral inequalities play a major role in the field of differential equations and applied mathematics. Applications of integral inequalities are found in applied sciences, such as statistical problems, transform theory, numerical quadrature, and probability. In the last few years, many researchers have established various types of integral inequalities by employing different approaches. The interested readers are suggested to see [9-11].

Moreover, the integral inequalities are linking with other areas such as differential equations, difference equations, mathematical analysis, mathematical physics, convexity theory, discrete fractional calculus, and fuzzy theory, see [12-19]. In the context of fractional 
calculus, the study of the integral operators taken to non integer orders [20,21], and most studies come about only in the real line.

Definition 1. Let $\psi$ be a function defined on a closed interval $\left[\xi_{1}, \xi_{2}\right]$. The left and right $R L$ fractional integrals of order $\alpha>0$ are given by

$$
\begin{array}{ll}
\left(\mathcal{I}_{\xi_{1}^{+}}^{\alpha} \psi\right)(x)=\frac{1}{\Gamma(\alpha)} \int_{\xi_{1}}^{x}(x-\tau)^{\alpha-1} \psi(\tau) d \tau & \left(x>\xi_{1}\right), \\
\left(\mathcal{I}_{\xi_{2}}^{\alpha} \psi\right)(x)=\frac{1}{\Gamma(\alpha)} \int_{x}^{\xi_{2}}(\tau-x)^{\alpha-1} \psi(\tau) d \tau & \left(x<\xi_{2}\right),
\end{array}
$$

respectively.

Fractional integral has been widely studied in the literature. The idea has been defined by many mathematicians with slightly different formulas, for example, RL, Weyl, Erdélyi-Kober, Hadamard integral, Liouville-Caputo and other fractional integrals [22].

One important type of integral inequalities consists of the familiar Chebyshev inequality, which is related to the synchronous functions. This has been intensively studied, with many book chapters and important research articles dedicated to the Chebyshev type inequalities, see [23-28]. We will develop in Section 4, some new results and basic examples as well using the same ideas as in recently published papers about certain generalized proportional fractional integrals from Rahman et al. (see [29-35]) in the framework of the new class of generalized fractional integral operators which will be defined at the end of Section 2.

The Chebyshev inequality is given as follows (see [25]):

$$
\frac{1}{\xi_{2}-\xi_{1}} \int_{\xi_{1}}^{\xi_{2}} \psi_{1}(\tau) \psi_{2}(\tau) \mathrm{d} \tau \geq\left(\frac{1}{\xi_{2}-\xi_{1}} \int_{\xi_{1}}^{\xi_{2}} \psi_{1}(\tau) \mathrm{d} \tau\right)\left(\frac{1}{\xi_{2}-\xi_{1}} \int_{\xi_{1}}^{\xi_{2}} \psi_{2}(\tau) \mathrm{d} \tau\right)
$$

where $\psi_{1}$ and $\psi_{2}$ are assumed to be integrable and synchronous functions on $\left[\xi_{1}, \xi_{2}\right]$. By definition, two functions are called synchronous on $\left[\xi_{1}, \xi_{2}\right]$ if the following inequality holds true:

$$
\left(\psi_{1}(x)-\psi_{1}(y)\right)\left(\psi_{2}(x)-\psi_{2}(y)\right) \geq 0
$$

for all $x, y \in\left[\xi_{1}, \xi_{2}\right]$.

In particular, the Chebyshev inequality (1) is useful due to its connections with fractional calculus, and it arises naturally in the existence of solutions to various integer-order or fractional-order differential equations, including some which are useful in practical applications such as those in numerical quadrature, transform theory, statistics, and probability, see [36-44].

There are many ways to define fractional derivatives and fractional integrals, often related to or inspired by the RL definitions (see, for example, [45-47]), regarding some general classes into which such fractional derivative and fractional integral operators can be classified. We always consider the most general possible setting in which a specific behaviour or result can be obtained in pure mathematics. However, it is important to consider particular types of fractional calculus suited to the models of given real-world problems in applied mathematics.

Some of these definitions of fractional calculus have properties that are from those of the standard RL definitions, and some of them can be used to the model of real-life data more effectively than the RL model, see [48-54]. As described in many recent articles cited herein, the fractional calculus definitions discussed in this article are useful, particularly in modelling real-world problems.

\section{Preliminaries}

Special functions have many relations with fractional calculus, see [20,55]. In particular, the ML type functions are remarkably significant in this area, see [56-61]. 
The familiar ML function $\mathcal{E}_{\alpha}(z)$ and its two-parameter version $\mathcal{E}_{\alpha, \beta}(z)$ are defined, respectively, by

$$
\mathcal{E}_{\alpha}(z):=\sum_{k=0}^{\infty} \frac{z^{k}}{\Gamma(\alpha k+1)} \quad \text { and } \quad \mathcal{E}_{\alpha, \beta}(z):=\sum_{k=0}^{\infty} \frac{z^{k}}{\Gamma(\alpha k+\beta)},
$$

where $z, \alpha, \beta \in \mathbb{C}$ and $\Re(\alpha)>0$, which were first considered by Magnus Gustaf (Gösta) Mittag-Leffler (1846-1927) in 1903 and Anders Wiman (1865-1959) in 1905.

In many recent investigations, the interest in the families of ML type functions has grown considerably due mainly to their potential for applications in some reaction-diffusion and other applied problems, and their various generalizations appear in the solutions of fractional-order differential and integral equations (see, for example, [62]). The following family of the multi-index ML functions:

$$
\mathcal{E}_{\gamma, \kappa, \epsilon}\left[\left(\alpha_{j}, \beta_{j}\right)_{j=1}^{m} ; z\right]
$$

was considered and used as a kernel of some fractional-calculus operators by Srivastava et al. (see $[63,64]$; see also the references cited in each of these papers):

$$
\begin{gathered}
\mathcal{E}_{\left(\alpha_{j}, \beta_{j}\right)_{m}}^{\gamma, \kappa, \varepsilon, \epsilon}[z]=\mathcal{E}_{\gamma, \kappa, \delta, \epsilon}\left[\left(\alpha_{j}, \beta_{j}\right)_{j=1}^{m} ; z\right]:=\sum_{n=0}^{\infty} \frac{(\gamma)_{\kappa n}(\delta)_{\epsilon n}}{\prod_{j=1}^{m} \Gamma\left(\alpha_{j} n+\beta_{j}\right)} \frac{z^{n}}{n !} \\
\left(\alpha_{j}, \beta_{j}, \gamma, \kappa, \delta, \epsilon \in \mathbb{C} ; \Re\left(\alpha_{j}\right)>0(j=1, \ldots, m) ; \Re\left(\sum_{j=1}^{m} \alpha_{j}\right)>\Re(\kappa+\epsilon)-1\right),
\end{gathered}
$$

where $(\lambda)_{v}$ denotes the general Pochhammer symbol or the shifted factorial, since

$$
(1)_{n}=n ! \quad\left(n \in \mathbb{N}_{0}:=\mathbb{N} \cup\{0\} ; \mathbb{N}:=\{1,2,3, \ldots\}\right),
$$

defined (for $\lambda, v \in \mathbb{C}$ and in terms of the familiar Gamma function) by

$$
(\lambda)_{v}:=\frac{\Gamma(\lambda+v)}{\Gamma(\lambda)}= \begin{cases}1, & (v=0 ; \lambda \in \mathbb{C} \backslash\{0\}) ; \\ \lambda(\lambda+1) \cdots(\lambda+n-1), & (v=n \in \mathbb{N} ; \lambda \in \mathbb{C}),\end{cases}
$$

it is assumed conventionally that $(0)_{0}:=1$ and understand tacitly that the $\Gamma$-quotient in (4) exists.

We now turn to the familiar FW hypergeometric function ${ }_{p} \Psi_{q}(z)$ (with $p$ numerator and $q$ denominator parameters), which is given by the following series (see Fox [65] and Wright [66,67]; see also ([5] [p. 67, Eq. 1.12(68)]) and ([68] [p. 21, Eq. 1.2(38)])):

$$
\begin{aligned}
{ }_{p} \Psi_{q}\left[\begin{array}{c}
\left(\alpha_{1}, \mathcal{P}_{1}\right), \ldots,\left(\alpha_{p}, \mathcal{P}_{p}\right) ; \\
\left(\beta_{1}, \mathcal{Q}_{1}\right), \ldots,\left(\beta_{q}, \mathcal{Q}_{q}\right) ;
\end{array}\right]:= & \sum_{n=0}^{\infty} \frac{\prod_{j=1}^{p} \Gamma\left(\alpha_{j}+\mathcal{P}_{j} n\right)}{\prod_{k=1}^{q} \Gamma\left(\beta_{k}+\mathcal{Q}_{k} n\right)} \frac{z^{n}}{n !} \\
& =\frac{\prod_{j=1}^{p} \Gamma\left(\alpha_{j}\right)}{\prod_{k=1}^{q} \Gamma\left(\beta_{k}\right)} \sum_{n=0}^{\infty} \frac{\prod_{j=1}^{p}\left(\alpha_{j}\right)_{\mathcal{P}_{j} n}}{\prod_{k=1}^{q}\left(\beta_{k}\right)_{\mathcal{Q}_{k} n}} \frac{z^{n}}{n !}
\end{aligned}
$$

in which we have made use of the general Pochhammer symbol $(\lambda)_{v}(\lambda, v \in \mathbb{C})$ defined by (4), the parameters

$$
\alpha_{j}, \beta_{k} \in \mathbb{C}(j=1, \ldots, p ; k=1, \ldots, q)
$$


and the coefficients

$$
\mathcal{P}_{1}, \ldots, \mathcal{P}_{p} \in \mathbb{R}^{+} \quad \text { and } \quad \mathcal{Q}_{1}, \ldots, \mathcal{Q}_{q} \in \mathbb{R}^{+}
$$

are so constrained that

$$
1+\sum_{k=1}^{q} \mathcal{Q}_{k}-\sum_{j=1}^{p} \mathcal{P}_{j} \geq 0
$$

with the equality for appropriately constrained values of the argument $z$. Thus, if we compare the definition (3) of the general multi-index ML function:

$$
\mathcal{E}_{\gamma, \kappa, \delta, \epsilon}\left[\left(\alpha_{j}, \beta_{j}\right)_{j=1}^{m} ; z\right]
$$

with the definition in (5), it immediately follows that

$$
\mathcal{E}_{\left(\alpha_{j}, \beta_{j}\right)_{m}}^{\gamma, \kappa, \epsilon, \epsilon}[z]=\mathcal{E}_{\gamma, \kappa, \delta, \epsilon}\left[\left(\alpha_{j}, \beta_{j}\right)_{j=1}^{m} ; z\right]=\frac{1}{\Gamma(\gamma) \Gamma(\delta)}{ }_{2} \Psi_{m}\left[\begin{array}{r}
(\gamma, \kappa),(\delta, \epsilon) ; \\
\left(\beta_{1}, \alpha_{1}\right), \ldots,\left(\beta_{m}, \alpha_{m}\right) ;
\end{array}\right] .
$$

We now recall a modified version $\mathcal{F}_{\rho, \lambda}^{\sigma}(z)$ of the FW function ${ }_{p} \Psi_{q}(z)$ in (5) as well as the ML type functions, which was introduced by Wright ([69] [p. 424]) in the year 1940, who partially and formally replaced the $\Gamma$-quotient in (5) by a sequence $\{\sigma(n)\}_{n=0}^{\infty}$ based upon a suitably-restricted function $\sigma(n)$ as follows:

$$
\mathcal{F}_{\rho, \lambda}^{\sigma}(z)=\mathcal{F}_{\rho, \lambda}^{\sigma(0), \sigma(1), \ldots}(z):=\sum_{n=0}^{\infty} \frac{\sigma(n)}{\Gamma(\rho n+\lambda)} z^{n}
$$

where $\rho, \lambda>0,|z|<R$, and $\{\sigma(n)\}_{n \in \mathbb{N}_{0}}$ is a bounded sequence in the real-number set $\mathbb{R}$. As already remarked in, for example, [70], this same function $\mathcal{F}_{\rho, \lambda}^{\sigma}$ was reproduced in [71], but without giving any credit to Wright [69]. In fact, in his recent survey-cum-expository review articles, the above-defined Wright function $\mathcal{F}_{\rho, \lambda}^{\sigma}$ in (8) as well as its well-motivated companions and extensions were used as the kernels in order to systematically study some general families of fractional-calculus (fractional integral and fractional derivative) operators by Srivastava (see, for details, [72]).

Definition 2 below makes a straightforward use of the Wright function $\mathcal{F}_{\rho, \lambda}^{\sigma}$ in the kernel of a family of fractional integral operators.

Definition 2 (see, for details, [70,72,73]). For a given $\mathcal{L}_{1}$-function $\psi$ on an interval $\left[\xi_{1}, \xi_{2}\right]$, the general left-side and right-side fractional integral operators, applied to a prescribed function $\psi(x)$, are defined for $\lambda, \rho>0$ and $w \in \mathbb{R}$ by

$$
\left(\mathcal{J}_{\rho, \lambda, \xi_{1}^{+} ; w}^{\sigma} \psi\right)(x)=\int_{\xi_{1}}^{x}(x-\xi)^{\lambda-1} \mathcal{F}_{\rho, \lambda}^{\sigma}\left[w(x-\xi)^{\rho}\right] \psi(\xi) \mathrm{d} \xi \quad\left(x>\xi_{1}\right)
$$

and

$$
\left(\mathcal{J}_{\rho, \lambda, \xi_{2}^{-} ; w}^{\sigma} \psi\right)(x)=\int_{x}^{\xi_{2}}(\xi-x)^{\lambda-1} \mathcal{F}_{\rho, \lambda}^{\sigma}\left[w(\xi-x)^{\rho}\right] \psi(\xi) \mathrm{d} \xi \quad\left(x<\xi_{2}\right),
$$

where the function $\psi$ is so constrained that the integrals on the right-hand sides exist and $\mathcal{F}_{\rho, \lambda}^{\sigma}$ is the Wright function defined by (8).

Remark 1. The function $\phi:[0, \infty) \rightarrow[0, \infty)$, which is constructed from the work of Sarikaya et al. (see [74]), has the following four conditions:

$$
\int_{0}^{1} \frac{\phi(\xi)}{\xi} d \xi<\infty,
$$




$$
\begin{gathered}
\frac{1}{\mathcal{A}_{1}} \leq \frac{\phi\left(\xi_{1}\right)}{\phi\left(\xi_{2}\right)} \leq \mathcal{A}_{1} \text { for } \frac{1}{2} \leq \frac{\xi_{1}}{\xi_{2}} \leq 2, \\
\frac{\phi\left(\xi_{2}\right)}{\xi_{2}^{2}} \leq \mathcal{A}_{2} \frac{\phi\left(\xi_{1}\right)}{\xi_{1}^{2}} \text { for } \xi_{1} \leq \xi_{2}
\end{gathered}
$$

and

$$
\left|\frac{\phi\left(\xi_{2}\right)}{\xi_{2}^{2}}-\frac{\phi\left(\xi_{1}\right)}{\xi_{1}^{2}}\right| \leq \mathcal{A}_{3}\left|\xi_{2}-\xi_{1}\right| \frac{\phi\left(\xi_{2}\right)}{\xi_{2}^{2}} \text { for } \frac{1}{2} \leq \frac{\xi_{1}}{\xi_{2}} \leq 2,
$$

where $\mathcal{A}_{1}, \mathcal{A}_{2}$ and $\mathcal{A}_{3}>0$ are independent of $\xi_{1}, \xi_{2}>0$. Moreover, Sarikaya et al. (see [74]) used the above function $\phi$ in order to define the following fractional integral operators.

Definition 3. The generalized left-side and right-side fractional integrals are given as follows:

$$
\xi_{1}^{+} \mathcal{I}_{\phi} \psi(x)=\int_{\xi_{1}}^{x} \frac{\phi(x-\xi)}{x-\xi} \psi(\xi) \mathrm{d} \xi \quad\left(x>\xi_{1}\right)
$$

and

$$
{ }_{\xi_{2}} \mathcal{I}_{\phi} \psi(x)=\int_{x}^{\xi_{2}} \frac{\phi(\xi-x)}{\xi-x} \psi(\xi) \mathrm{d} \xi \quad\left(x<\xi_{2}\right),
$$

respectively.

Furthermore, Sarikaya et al. [74] noticed that the generalized fractional integrals given by Definition 3 may contain some types of fractional integrals such as the RL and other fractional integrals for some special choices of function $\phi$.

Inspired by the above definitions and related developments, we are able here to define and investigate a new family of generalized fractional integral operators involving the Wright function $\mathcal{F}_{\rho, \lambda}^{\sigma}$ defined by (8).

Definition 4. For a given $\mathcal{L}_{1}$-function $\psi$ on an interval $\left[\xi_{1}, \xi_{2}\right]$, the generalized left-side and right-side fractional integral operators, applied to $\psi(x)$, are defined for $\lambda, \rho>0$ and $w \in \mathbb{R}$ by

$$
\left(\mathcal{T}_{\sigma, \rho, \lambda, \xi_{1}^{+} ; w}^{\phi} \psi\right)(x)=\int_{\xi_{1}}^{x} \frac{\phi(x-\xi)}{x-\xi} \mathcal{F}_{\rho, \lambda}^{\sigma}\left[w(x-\xi)^{\rho}\right] \psi(\xi) \mathrm{d} \xi \quad\left(x>\xi_{1}\right)
$$

and

$$
\left(\mathcal{T}_{\sigma, \rho, \lambda, \xi_{2}^{-} ; w}^{\phi} \psi\right)(x)=\int_{x}^{\xi_{2}} \frac{\phi(\xi-x)}{\xi-x} \mathcal{F}_{\rho, \lambda}^{\sigma}\left[w(\xi-x)^{\rho}\right] \psi(\xi) \mathrm{d} \xi \quad\left(x<\xi_{2}\right),
$$

where the function $\psi$ is so constrained that the integrals on the right-hand sides exist and $\mathcal{F}_{\rho, \lambda}^{\sigma}$ is the modified ML function.

Remark 2. Each of the following special cases is worthy of note:

- $\quad$ Taking $\phi(\xi)=\frac{\xi^{\alpha}}{\Gamma(\alpha)}$ with $\lambda=\sigma(0)=1$ and $\sigma(n)=0$ for all $n \neq 0$, and $w=0$ in our definition, then we have Definition 1.

- $\quad$ Choosing $\phi(\xi)=\xi^{\lambda}$ in our definition, then we get Definition 2 .

- Setting $\lambda=\sigma(0)=1$ and $\sigma(n)=0$ for all $n \neq 0$, and $w=0$ in our definition, then we obtain Definition 3.

Remark 3. Two important special cases of our Definition 4 are given as follows:

(I) Taking $\phi(\xi)=\xi\left(\xi_{2}-\xi\right)^{\alpha-1}$ for all $\xi \in\left[\xi_{1}, \xi_{2}\right]$ and $\alpha \in(0,1]$, we have the so-called conformable left-side and right-side fractional integral operators defined by

$$
\left(\mathcal{C}_{\sigma, \rho, \lambda, \xi_{1}^{+} ; w}^{\alpha} \psi\right)(x)=\int_{\xi_{1}}^{x}\left(\xi+\xi_{2}-x\right)^{\alpha-1} \mathcal{F}_{\rho, \lambda}^{\sigma}\left[w(x-\xi)^{\rho}\right] \psi(\xi) \mathrm{d} \xi \quad\left(x>\xi_{1}\right)
$$


and

$$
\left(\mathcal{C}_{\sigma, \rho, \lambda, \xi_{2} ; \tau^{-}}^{\alpha} \psi\right)(x)=\int_{x}^{\xi_{2}}\left(x+\xi_{2}-\xi\right)^{\alpha-1} \mathcal{F}_{\rho, \lambda}^{\sigma}\left[w(\xi-x)^{\rho}\right] \psi(\xi) \mathrm{d} \xi \quad\left(x<\xi_{2}\right) .
$$

(II) Choosing

$$
\phi(\xi)=\frac{\xi}{\alpha} \exp (-\mathcal{A} \xi)
$$

where

$$
\mathcal{A}=\frac{1-\alpha}{\alpha}
$$

and $\alpha \in(0,1]$ for all $\xi \in\left[\xi_{1}, \xi_{2}\right]$, we get the so-called exponential left-side and right-side fractional integral operators defined by

$$
\begin{aligned}
& \left(\mathcal{E}_{\sigma, \rho, \lambda, \xi_{1}^{+} ; w}^{\alpha} \psi\right)(x) \\
& =\frac{1}{\alpha} \int_{\xi_{1}}^{x} \exp (-\mathcal{A}(x-\xi)) \mathcal{F}_{\rho, \lambda}^{\sigma}\left[w(x-\xi)^{\rho}\right] \psi(\xi) \mathrm{d} \xi \quad\left(x>\xi_{1}\right)
\end{aligned}
$$

and

$$
\begin{aligned}
& \left(\mathcal{E}_{\sigma, \rho, \lambda, \xi_{2}^{-} ; w}^{\alpha} \psi\right)(x) \\
& =\frac{1}{\alpha} \int_{x}^{\tilde{\xi}_{2}} \exp (-\mathcal{A}(\xi-x)) \mathcal{F}_{\rho, \lambda}^{\sigma}\left[w(\xi-x)^{\rho}\right] \psi(\xi) \mathrm{d} \xi \quad\left(x<\xi_{2}\right) .
\end{aligned}
$$

This paper is organized as follows: In Section 3, we will introduce the generalized left-side and right-side fractional integral operators with a certain modified ML kernel. We will investigate the Chebyshev inequality via this general family of fractional integral operators. Moreover, we derive new results of this type inequality for the finite product of functions. In addition, we will establish an estimate for the Chebyshev functional by using the new fractional integral operators. Some special cases will be derived in details from our results. In Section 4, two important results and some interesting consequences for convex functions in the framework of the defined class of generalized fractional integral operators will be established. Furthermore, two basic examples demonstrated the significance of our new results in this section. Finally, we give the conclusions in Section 5.

\section{Main Results and Their Consequences}

Throughout our study, we suppose that $\{\sigma(n)\}_{n \in \mathbb{N}_{0}}$ is a sequence of non-negative real numbers and the function $\phi:[0, \infty) \rightarrow[0, \infty)$ satisfies the conditions (11)-(14). Our main results are given below.

Theorem 1. Let $\lambda, \rho>0$ and $w \in \mathbb{R}$. Assume that $\psi_{1}$ and $\psi_{2}$ are two synchronous functions on $\left[\xi_{1}, \infty\right)$. Then

$$
\begin{aligned}
& \left(\mathcal{T}_{\sigma, \rho, \lambda, \xi_{1}^{+} ; w}^{\phi} \psi_{1} \psi_{2}\right)(\xi) \\
& \quad \geq \frac{1}{\left(\mathcal{T}_{\sigma, \rho, \lambda, \xi_{1}^{+} ; w}^{\phi} 1\right)(\xi)}\left(\mathcal{T}_{\sigma, \rho, \lambda, \xi_{1}^{+} ; w^{\prime}}^{\phi} \psi_{1}\right)(\xi)\left(\mathcal{T}_{\sigma, \rho, \lambda, \xi_{1}^{+} ; w^{\prime}}^{\phi} \psi_{2}\right)(\xi) \quad\left(\forall \xi>\xi_{1} \geq 0\right) .
\end{aligned}
$$

Proof. Since the functions $\psi_{1}$ and $\psi_{2}$ are synchronous on $\left[\xi_{1}, \infty\right)$, we find for $r, s \geq \xi_{1}$ that

$$
\left(\psi_{1}(r)-\psi_{1}(s)\right)\left(\psi_{2}(r)-\psi_{2}(s)\right) \geq 0 .
$$


It follows that

$$
\psi_{1}(r) \psi_{2}(r)+\psi_{1}(s) \psi_{2}(s) \geq \psi_{1}(r) \psi_{2}(s)+\psi_{1}(s) \psi_{2}(r) .
$$

By multiplying both sides of (23) by

$$
\frac{\phi(\xi-r)}{\xi-r} \mathcal{F}_{\rho, \lambda}^{\sigma}\left[w(\xi-r)^{\rho}\right]
$$

with $r \in\left(\xi_{1}, \xi\right)$, we can deduce that

$$
\begin{aligned}
& \frac{\phi(\xi-r)}{\xi-r} \mathcal{F}_{\rho, \lambda}^{\sigma}\left[w(\xi-r)^{\rho}\right] \psi_{1}(r) \psi_{2}(r)+\frac{\phi(\xi-r)}{\xi-r} \mathcal{F}_{\rho, \lambda}^{\sigma}\left[w(\xi-r)^{\rho}\right] \psi_{1}(s) \psi_{2}(s) \\
& \quad \geq \frac{\phi(\xi-r)}{\xi-r} \mathcal{F}_{\rho, \lambda}^{\sigma}\left[w(\xi-r)^{\rho}\right] \psi_{1}(r) \psi_{2}(s)+\frac{\phi(\xi-r)}{\xi-r} \mathcal{F}_{\rho, \lambda}^{\sigma}\left[w(\xi-r)^{\rho}\right] \psi_{1}(s) \psi_{2}(r),
\end{aligned}
$$

which, upon integration over $r \in\left(\xi_{1}, \xi\right)$, yields

$$
\begin{aligned}
& \left(\mathcal{T}_{\sigma, \rho, \lambda, \xi_{1}^{+} ; w}^{\phi} \psi_{1} \psi_{2}\right)(\xi)+\psi_{1}(s) \psi_{2}(s)\left(\mathcal{T}_{\sigma, \rho, \lambda, \xi_{1}^{+} ; w^{\prime}}^{\phi}\right)(\xi) \\
& \quad \geq \psi_{2}(s)\left(\mathcal{T}_{\sigma, \rho, \lambda, \xi_{1}^{+} ; w}^{\phi} \psi_{1}\right)(\xi)+\psi_{1}(s)\left(\mathcal{T}_{\sigma, \rho, \lambda, \xi_{1}^{+} ; w}^{\phi} \psi_{2}\right)(\xi)
\end{aligned}
$$

Now, by applying symmetry considerations with respect to other variable $s \in\left(\xi_{1}, \xi\right)$ and using the same technique as above, we complete the proof of Theorem 1.

Remark 4. If we take $\phi(\xi)=\xi^{\lambda}$ in Theorem 1, we obtain ([39] Theorem 2) or ([40] Corollary 3.11).

We next state and prove Theorem 2 below.

Theorem 2. Let $\lambda, \rho>0$ and $w \in \mathbb{R}$. Also, let $\left\{\psi_{i}\right\}_{i=1}^{n}$ be $n$ positive and increasing functions defined on $\left[\xi_{1}, \infty\right)$. Then

$$
\begin{aligned}
\left(\mathcal{T}_{\sigma, \rho, \lambda, \xi_{1}^{+} ; w}^{\phi}\right. & \left.\prod_{i=1}^{n} \psi_{i}\right)(\xi) \\
& \geq\left[\frac{1}{\left(\mathcal{T}_{\sigma, \rho, \lambda, \xi_{1}^{+} ; w^{\prime}}^{\phi}\right)(\xi)}\right]^{n-1} \prod_{i=1}^{n}\left(\mathcal{T}_{\sigma, \rho, \lambda, \xi_{1}^{+} ; w}^{\phi} \psi_{i}\right)(\xi) \quad\left(\forall \xi>\xi_{1} \geq 0\right) .
\end{aligned}
$$

Proof. The proof will make use of the principle of mathematical induction. Firstly, for $n=1$, we have

$$
\left(\mathcal{T}_{\sigma, \rho, \lambda, \xi_{1}^{+} ; \omega}^{\phi} \psi_{1}\right)(\xi) \geq\left(\mathcal{T}_{\sigma, \rho, \lambda, \xi_{1}^{+} ; \omega}^{\phi} \psi_{1}\right)(\xi) \quad\left(\forall \xi>\xi_{1} \geq 0\right) .
$$

In the case when $n=2$, since $\psi_{1}$ and $\psi_{2}$ are increasing functions defined on $\left[\xi_{1}, \infty\right)$, then from Theorem 1, we have

$$
\begin{aligned}
& \left(\mathcal{T}_{\sigma, \rho, \lambda, \xi_{1}^{+} ; w}^{\phi} \psi_{1} \psi_{2}\right)(\xi) \\
& \quad \geq \frac{1}{\left(\mathcal{T}_{\sigma, \rho, \lambda, \xi_{1}^{+} ; w^{+}}^{\phi} 1\right)(\xi)}\left(\mathcal{T}_{\sigma, \rho, \lambda, \xi_{1}^{+} ; w^{\prime}}^{\phi} \psi_{1}\right)(\xi)\left(\mathcal{T}_{\sigma, \rho, \lambda, \xi_{1}^{+} ; w}^{\phi} \psi_{2}\right)(\xi) \quad\left(\forall \xi>\xi_{1} \geq 0\right) .
\end{aligned}
$$


We now assume that the inequality (24) holds true for some $n \in \mathbb{N}$. Then, since the $n$ functions $\left\{\psi_{i}\right\}_{i=1}^{n}$ are positive and increasing on $\left[\xi_{1}, \infty\right), \prod_{i=1}^{n} \psi_{i}$ is also an increasing function. Hence, we can apply Theorem 1 with

$$
\psi_{1}{ }^{*}:=\prod_{i=1}^{n-1} \psi_{i} \quad \text { and } \quad \psi_{2}{ }^{*}:=\psi_{n}
$$

in order to obtain

$$
\begin{aligned}
\left(\mathcal{T}_{\sigma, \rho, \lambda, \xi_{1}^{+} ; w}^{\phi} \prod_{i=1}^{n} \psi_{i}\right)(\xi) & =\left(\mathcal{T}_{\sigma, \rho, \lambda, \xi_{1}^{+} ; w}^{\phi} \psi_{1}{ }^{*} \psi_{2}{ }^{*}\right)(\xi) \\
& \geq \frac{1}{\left(\mathcal{T}_{\sigma, \rho, \lambda, \xi_{1}^{+} ; w}^{\phi} 1\right)(\xi)}\left(\mathcal{T}_{\sigma, \rho, \lambda, \xi_{1}^{+} ; w^{\phi}}^{\phi} \psi_{1}^{*}\right)(\xi)\left(\mathcal{T}_{\sigma, \rho, \lambda, \xi_{1}^{+} ; w^{\phi}}^{\phi} \psi_{2}^{*}\right)(\xi) \\
& =\frac{1}{\left(\mathcal{T}_{\sigma, \rho, \lambda, \xi_{1}^{+} ; w^{\prime}}^{\phi} 1\right)(\xi)}\left(\mathcal{T}_{\sigma, \rho, \lambda, \xi_{1}^{+} ; w}^{\phi} \prod_{i=1}^{n-1} \psi_{i}\right)(\xi)\left(\mathcal{T}_{\sigma, \rho, \lambda, \xi_{1}^{+} ; w}^{\phi} \psi_{n}\right)(\xi)
\end{aligned}
$$

Thus, if we make use of our assumed inequality (24) in the last inequality, we have

$$
\begin{aligned}
\left(\mathcal{T}_{\sigma, \rho, \lambda, \xi_{1}^{+} ; w}^{\phi} \prod_{i=1}^{n} \psi_{i}\right)(\xi) & \geq\left[\frac{1}{\left(\mathcal{T}_{\sigma, \rho, \lambda, \xi_{1}^{+} ; w}^{\phi} 1\right)(\xi)}\right]\left[\frac{1}{\left(\mathcal{T}_{\sigma, \rho, \lambda, \xi_{1}^{+} ; w}^{\phi} 1\right)(\xi)}\right]^{(n-1)-1} \\
& \times \prod_{i=1}^{n-1}\left(\mathcal{T}_{\sigma, \rho, \lambda, \xi_{1}^{+} ; w}^{\phi} \psi_{i}\right)(\xi)\left(\mathcal{T}_{\sigma, \rho, \lambda, \xi_{1}^{+} ; w}^{\phi} \psi_{n}\right)(\xi) \\
& =\left[\frac{1}{\left(\mathcal{T}_{\sigma, \rho, \lambda, \xi_{1}^{+} ; w^{\prime}}^{\phi}\right)(\xi)}\right]^{n-1} \prod_{i=1}^{n}\left(\mathcal{T}_{\sigma, \rho, \lambda, \xi_{1}^{+} ; w}^{\phi} \psi_{i}\right)(\xi) .
\end{aligned}
$$

This completes the proof of Theorem 2.

Remark 5. If we set $\phi(\xi)=\xi^{\lambda}$ in Theorem 2, we obtain ([39] Theorem 4).

We next state and prove Theorem 3 below.

Theorem 3. Let $\lambda, \rho>0$ and $w \in \mathbb{R}$. Also let $\psi_{1}, \psi_{2}$ be two functions such that the function $\psi_{1}$ is increasing and the function $\psi_{2}$ is differentiable. If there exists a real number $\mathfrak{m}$ with $\mathfrak{m}:=$ $\inf _{\tilde{\zeta} \geq 0} \psi_{2}^{\prime}(\xi)$, then

$$
\begin{aligned}
&\left(\mathcal{T}_{\sigma, \rho, \lambda, \xi_{1}^{+} ; w}^{\phi} \psi_{1} \psi_{2}\right)(\xi) \geq \frac{1}{\left(\mathcal{T}_{\sigma, \rho, \lambda, \xi_{1}^{+} ; w}^{\phi} 1\right)(\xi)}\left(\mathcal{T}_{\sigma, \rho, \lambda, \xi_{1}^{+} ; w}^{\phi} \psi_{1}\right)(\xi)\left(\mathcal{T}_{\sigma, \rho, \lambda, \xi_{1}^{+} ; w}^{\phi} \psi_{2}\right)(\xi) \\
&-\frac{\mathfrak{m}}{\left(\mathcal{T}_{\sigma, \rho, \lambda, \xi_{1}^{+} ; w^{+}}^{\phi} 1\right)(\xi)}\left(\mathcal{T}_{\sigma, \rho, \lambda, \xi_{1}^{+} ; w}^{\phi} \psi_{1}\right)(\xi)\left(\mathcal{T}_{\sigma, \rho, \lambda, \xi_{1}^{+} ; w}^{\phi} \operatorname{Id}\right)(\xi) \\
&+\mathfrak{m}\left(\mathcal{T}_{\sigma, \rho, \lambda, \xi_{1}^{+} ; w}^{\phi} \operatorname{Id} \cdot \psi_{1}\right)(\xi)
\end{aligned}
$$

where

$$
\operatorname{Id}(\xi):=\xi \quad\left(\forall \xi>\xi_{1} \geq 0\right) .
$$


Proof. Let us define the following function:

$$
\hbar(\xi):=\psi_{2}(\xi)-\mathfrak{m} \operatorname{Id}(\xi)
$$

where $\operatorname{Id}(\xi):=\xi$. One can easily verify that $h$ is an increasing and differentiable function on $\left[\xi_{1}, \infty\right)$. Then, by using Theorem 1 , we have

$$
\begin{gathered}
\left(\mathcal{T}_{\sigma, \rho, \lambda, \xi_{1}^{+} ; w}^{\phi} \psi_{1} \hbar\right)(\xi) \geq \frac{1}{\left(\mathcal{T}_{\sigma, \rho, \lambda, \xi_{1}^{+} ; w}^{\phi} 1\right)(\xi)}\left(\mathcal{T}_{\sigma, \rho, \lambda, \xi_{1}^{+} ; w}^{\phi} \psi_{1}\right)(\xi)\left(\mathcal{T}_{\sigma, \rho, \lambda, \xi_{1}^{+} ; w}^{\phi} h\right)(\xi) \\
=\frac{1}{\left(\mathcal{T}_{\sigma, \rho, \lambda, \xi_{1}^{+} ; w^{\prime}}^{\phi}\right)(\xi)}\left(\mathcal{T}_{\sigma, \rho, \lambda, \xi_{1}^{+} ; w^{\prime}}^{\phi} \psi_{1}\right)(\xi)\left[\left(\mathcal{T}_{\sigma, \rho, \lambda, \xi_{1}^{+} ; w^{\phi}}^{\phi} \psi_{2}\right)(\xi)\right. \\
\left.-\mathfrak{m}\left(\mathcal{T}_{\sigma, \rho, \lambda, \xi_{1}^{+} ; w}^{\phi} \operatorname{Id}\right)(\xi)\right] .
\end{gathered}
$$

Moreover, since

$$
\left(\mathcal{T}_{\sigma, \rho, \lambda, \xi_{1}^{+} ; w}^{\phi} \psi_{1} \hbar\right)(\xi)=\left(\mathcal{T}_{\sigma, \rho, \lambda, \xi_{1}^{+} ; \omega}^{\phi} \psi_{1} \psi_{2}\right)(\xi)-\mathfrak{m}\left(\mathcal{T}_{\sigma, \rho, \lambda, \xi_{1}^{+} ; w}^{\phi} \operatorname{Id} \cdot \psi_{1}\right)(\xi),
$$

it follows that

$$
\begin{gathered}
\left(\mathcal{T}_{\sigma, \rho, \lambda, \xi_{1}^{+} ; w}^{\phi} \psi_{1} \psi_{2}\right)(\xi) \geq \frac{1}{\left(\mathcal{T}_{\sigma, \rho, \lambda, \xi_{1}^{+} ; w^{\prime}}^{\phi}\right)(\xi)}\left(\mathcal{T}_{\sigma, \rho, \lambda, \xi_{1}^{+} ; w}^{\phi} \psi_{1}\right)(\xi)\left(\mathcal{T}_{\sigma, \rho, \lambda, \xi_{1}^{+} ; w}^{\phi} \psi_{2}\right)(\xi) \\
-\frac{\mathfrak{m}}{\left(\mathcal{T}_{\sigma, \rho, \lambda, \xi_{1}^{+} ; w^{\prime}}^{\phi}\right)(\xi)}\left(\mathcal{T}_{\sigma, \rho, \lambda, \xi_{1}^{+} ; w}^{\phi} \psi_{1}\right)(\xi)\left(\mathcal{T}_{\sigma, \rho, \lambda, \xi_{1}^{+} ; w}^{\phi} \operatorname{Id}\right)(\xi) \\
+\mathfrak{m}\left(\mathcal{T}_{\sigma, \rho, \lambda, \xi_{1}^{+} ; w}^{\phi} \operatorname{Id} \cdot \psi_{1}\right)(\xi)
\end{gathered}
$$

This evidently completes the proof of Theorem 3.

Remark 6. Upon setting $\phi(\xi)=\xi^{\lambda}$ in Theorem 3, we obtain ([39] Theorem 5).

Let us discuss some important special cases and consequences of Theorem 3 below.

Corollary 1. Let $\lambda, \rho>0$ and $w \in \mathbb{R}$. Also let $\psi_{1}$ and $\psi_{2}$ be two functions such that $\psi_{1}$ is increasing and $\psi_{2}$ is differentiable. If there is a real number $M$ with $M:=\sup _{\xi \geq 0} \psi_{2}{ }^{\prime}(\xi)$, then the following inequality:

$$
\begin{gathered}
\left(\mathcal{T}_{\sigma, \rho, \lambda, \xi_{1}^{+} ; w}^{\phi} \psi_{1} \psi_{2}\right)(\xi) \geq \frac{1}{\left(\mathcal{T}_{\sigma, \rho, \lambda, \xi_{1}^{+} ; w}^{\phi} 1\right)(\xi)}\left(\mathcal{T}_{\sigma, \rho, \lambda, \xi_{1}^{+} ; w}^{\phi} \psi_{1}\right)(\xi)\left(\mathcal{T}_{\sigma, \rho, \lambda, \xi_{1}^{+} ; w}^{\phi} \psi_{2}\right)(\xi) \\
-\frac{M}{\left(\mathcal{T}_{\sigma, \rho, \lambda, \xi_{1}^{+} ; w^{\prime}}^{\phi}\right)(\xi)}\left(\mathcal{T}_{\sigma, \rho, \lambda, \xi_{1}^{+} ; w}^{\phi} \psi_{1}\right)(\xi)\left(\mathcal{T}_{\sigma, \rho, \lambda, \xi_{1}^{+} ; w}^{\phi} \mathrm{Id}\right)(\xi) \\
+M\left(\mathcal{T}_{\sigma, \rho, \lambda, \xi_{1}^{+} ; w}^{\phi} \mathrm{Id} \cdot \psi_{1}\right)(\xi)
\end{gathered}
$$

holds true for all $\xi>\xi_{1} \geq 0$. 
Proof. By the same technique as that used for proving Theorem 3, together with

$$
\hbar(\xi):=\psi_{2}(\xi)-M \operatorname{Id}(\xi),
$$

we can obtain the desired result asserted by Corollary 1.

Corollary 2. Let $\lambda, \rho>0$ and $w \in \mathbb{R}$. Also let $\psi_{1}$ and $\psi_{2}$ be two functions such that $\psi_{1}$ is increasing and both $\psi_{1}$ and $\psi_{2}$ are differentiable. If there exist real numbers $\mathfrak{m}_{1}$ and $\mathfrak{m}_{2}$ with

$$
\mathfrak{m}_{1}:=\inf _{\xi \geq 0} \psi_{1}{ }^{\prime}(\xi) \quad \text { and } \quad \mathfrak{m}_{2}:=\inf _{\xi \geq 0} \psi_{2}{ }^{\prime}(\xi)
$$

then the following inequality:

$$
\begin{aligned}
&\left(\mathcal{T}_{\sigma, \rho, \lambda, \xi_{1}^{+} ; w}^{\phi} \psi_{1} \psi_{2}\right)(\xi)-\mathfrak{m}_{1}\left(\mathcal{T}_{\sigma, \rho, \lambda, \xi_{1}^{+} ; w}^{\phi} \operatorname{Id} \cdot \psi_{2}\right)(\xi)-\mathfrak{m}_{2}\left(\mathcal{T}_{\sigma, \rho, \lambda, \xi_{1}^{+} ; w}^{\phi} \mathrm{Id} \cdot \psi_{1}\right)(\xi)+\mathfrak{m}_{1} \mathfrak{m}_{2}\left(\mathcal{T}_{\sigma, \rho, \lambda, \xi_{1}^{+} ; w}^{\phi} \mathrm{Id}^{2}\right)(\xi) \\
& \geq \frac{1}{\left(\mathcal{T}_{\sigma, \rho, \lambda, \xi_{1}^{+} ; w}^{\phi} 1\right)(\xi)}\left[\left(\mathcal{T}_{\sigma, \rho, \lambda, \xi_{1}^{+} ; w}^{\phi} \psi_{1}\right)(\xi)\left(\mathcal{T}_{\sigma, \rho, \lambda, \xi_{1}^{+} ; w}^{\phi} \psi_{2}\right)(\xi)\right. \\
&-\mathfrak{m}_{1}\left(\mathcal{T}_{\sigma, \rho, \lambda, \xi_{1}^{+} ; w}^{\phi} \mathrm{Id}\right)(\xi)\left(\mathcal{T}_{\sigma, \rho, \lambda, \xi_{1}^{+} ; w}^{\phi} \psi_{1}\right)(\xi) \\
&\left.-\mathfrak{m}_{2}\left(\mathcal{T}_{\sigma, \rho, \lambda, \xi_{1}^{+} ; w}^{\phi} \mathrm{Id}\right)(\xi)\left(\mathcal{T}_{\sigma, \rho, \lambda, \xi_{1}^{+} ; w}^{\phi} \psi_{2}\right)(\xi)+\mathfrak{m}_{1} \mathfrak{m}_{2}\left[\left(\mathcal{T}_{\sigma, \rho, \lambda, \xi_{1}^{+} ; w}^{\phi} \mathrm{Id}\right)(\xi)\right]^{2}\right]
\end{aligned}
$$

holds true for all $\xi>\xi_{1} \geq 0$.

Proof. By the same technique used for Theorem 3 with the setting

$$
\hbar_{1}(\xi):=\psi_{2}(\xi)-\mathfrak{m}_{1} \operatorname{Id}(\xi) \quad \text { and } \quad \hbar_{2}(\xi):=\psi_{2}(\xi)-\mathfrak{m}_{2} \operatorname{Id}(\xi),
$$

we can obtain the desired result asserted by Corollary 2 .

Corollary 3. Let $\lambda, \rho>0$ and $w \in \mathbb{R}$. Also let $\psi_{1}$ and $\psi_{2}$ be two functions such that $\psi_{1}$ is increasing and both $\psi_{1}$ and $\psi_{2}$ are differentiable. If there exist real numbers

$$
M_{1}:=\sup _{\xi \geq 0} \psi_{1}{ }^{\prime}(\xi) \quad \text { and } \quad M_{2}:=\sup _{\xi \geq 0} \psi_{2}{ }^{\prime}(\xi),
$$

then the following inequality:

$$
\begin{aligned}
& \left(\mathcal{T}_{\sigma, \rho, \lambda, \xi_{1}^{+} ; w}^{\phi} \psi_{1} \psi_{2}\right)(\xi)-M_{1}\left(\mathcal{T}_{\sigma, \rho, \lambda, \xi_{1}^{+} ; w}^{\phi} \mathrm{Id} \cdot \psi_{2}\right)(\xi) \\
& -M_{2}\left(\mathcal{T}_{\sigma, \rho, \lambda, \xi_{1}^{+} ; w}^{\phi} \operatorname{Id} \cdot \psi_{1}\right)(\xi)+M_{1} M_{2}\left(\mathcal{T}_{\sigma, \rho, \lambda, \xi_{1}^{+} ; w^{\prime}}^{\phi} \operatorname{Id}^{2}\right)(\xi) \\
& \geq \frac{1}{\left(\mathcal{T}_{\sigma, \rho, \lambda, \xi_{1}^{+} ; w^{\prime}}^{\phi} 1\right)(\xi)}\left[\left(\mathcal{T}_{\sigma, \rho, \lambda, \xi_{1}^{+} ; w}^{\phi} \psi_{1}\right)(\xi)\left(\mathcal{T}_{\sigma, \rho, \lambda, \xi_{1}^{+} ; w}^{\phi} \psi_{2}\right)(\xi)\right. \\
& -M_{1}\left(\mathcal{T}_{\sigma, \rho, \lambda, \xi_{1}^{+} ; w}^{\operatorname{Id}}\right)(\xi)\left(\mathcal{T}_{\sigma, \rho, \lambda, \xi_{1}^{+} ; w}^{\phi} \psi_{1}\right)(\xi) \\
& \left.-M_{2}\left(\mathcal{T}_{\sigma, \rho, \lambda, \xi_{1}^{+} ; w}^{\phi} \mathrm{Id}\right)(\xi)\left(\mathcal{T}_{\sigma, \rho, \lambda, \xi_{1}^{+} ; w}^{\phi} \psi_{2}\right)(\xi)+M_{1} M_{2}\left[\left(\mathcal{T}_{\sigma, \rho, \lambda, \xi_{1}^{+} ; w}^{\phi} \mathrm{Id}\right)(\xi)\right]^{2}\right]
\end{aligned}
$$


holds true for all $\xi>\xi_{1} \geq 0$.

Proof. By applying the same technique used for proving Theorem 3 with the setting

$$
\hbar_{1}(\xi):=\psi_{2}(\xi)-M_{1} \operatorname{Id}(\xi) \quad \text { and } \quad \hbar_{2}(\xi):=\psi_{2}(\xi)-M_{2} \operatorname{Id}(\xi),
$$

we can derive the desired result asserted by Corollary 3 .

Theorem 4. Let $\lambda, \rho>0$ and $w \in \mathbb{R}$. Also let $\hbar$ be a positive function on $[0, \infty)$ and suppose that $\psi_{1}$ and $\psi_{2}$ are two differentiable functions on $[0, \infty)$. If $\psi_{1}{ }^{\prime} \in \mathcal{L}_{r}[0, \infty)$ and $\psi_{2}{ }^{\prime} \in \mathcal{L}_{s}[0, \infty)$ with $r>1$ and $r^{-1}+s^{-1}=1$, then

$$
\begin{aligned}
& 2 \mid\left(\mathcal{T}_{\sigma, \rho, \lambda, 0^{+} ; w}^{\phi} \hbar \psi_{1} \psi_{2}\right)(\xi)\left(\mathcal{T}_{\sigma, \rho, \lambda, 0^{+} ; w}^{\phi} \hbar\right)(\xi)-\left(\mathcal{T}_{\sigma, \rho, \lambda, 0^{+} ; w}^{\phi} \hbar \psi_{1}\right)(\xi)\left(\mathcal{T}_{\sigma, \rho, \lambda, 0^{+} ; w}^{\phi} \hbar \psi_{2}\right)(\xi) \mid \\
& \leq\left\|\psi_{1}{ }^{\prime}\right\|_{r} \cdot\left\|\psi_{2}{ }^{\prime}\right\|_{s} \cdot \xi \int_{0}^{\xi} \int_{0}^{\tau} \frac{\phi(\xi-v)}{\xi-v} \frac{\phi(\xi-\tau)}{\xi-\tau} \mathcal{F}_{\rho, \lambda}^{\sigma}\left[w(\xi-v)^{\rho}\right] \\
& \times \mathcal{F}_{\rho, \lambda}^{\sigma}\left[w(\xi-\tau)^{\rho}\right] \hbar(v) \hbar(\tau) \mathrm{d} \tau \mathrm{d} v \\
& \leq\left\|\psi_{1}{ }^{\prime}\right\|_{r} \cdot\left\|\psi_{2}\right\|_{s} \cdot \xi\left[\left(\mathcal{T}_{\sigma, \rho, \lambda, 0^{+} ; w}^{\phi} \hbar\right)(\xi)\right]^{2} .
\end{aligned}
$$

Proof. Let $\hbar, \psi_{1}$ and $\psi_{2}$ be three functions that fulfill the hypotheses of Theorem 4 . We define

$$
\mathcal{H}(\tau, v):=\left(\psi_{1}(\tau)-\psi_{1}(v)\right)\left(\psi_{2}(\tau)-\psi_{2}(v)\right) \quad(\tau, v \in(0, \xi) ; \xi>0) .
$$

If we first multiply (26) by

$$
\frac{\phi(\xi-\tau)}{\xi-\tau} \mathcal{F}_{\rho, \lambda}^{\sigma}\left[w(\xi-\tau)^{\rho}\right] \hbar(\tau)
$$

with $\tau \in(0, \xi)$, and then integrate over $\tau \in(0, \xi)$, we get

$$
\begin{aligned}
\int_{0}^{\xi} \frac{\phi(\xi-\tau)}{\xi-\tau} \mathcal{F}_{\rho, \lambda}^{\sigma}\left[w(\xi-\tau)^{\rho}\right] \hbar(\tau) \mathcal{H}(\tau, v) \mathrm{d} \tau \\
=\left(\mathcal{T}_{\sigma, \rho, \lambda, 0^{+} ; w}^{\phi} \hbar \psi_{1} \psi_{2}\right)(\xi)-\psi_{1}(v)\left(\mathcal{T}_{\sigma, \rho, \lambda, 0^{+} ; w}^{\phi} \hbar \psi_{2}\right)(\xi) \\
\quad \quad-\psi_{2}(v)\left(\mathcal{T}_{\sigma, \rho, \lambda, 0^{+} ; w}^{\phi} \hbar \psi_{1}\right)(\xi)+\psi_{1}(v) \psi_{2}(v)\left(\mathcal{T}_{\sigma, \rho, \lambda, 0^{+} ; w^{\prime}}^{\phi} \hbar\right)(\xi) .
\end{aligned}
$$

We now multiply both sides of (27) by

$$
\frac{\phi(\xi-v)}{\xi-v} \mathcal{F}_{\rho, \lambda}^{\sigma}\left[w(\xi-v)^{\rho}\right] \hbar(v)
$$

with $v \in(0, \xi)$, and then integrate over $v \in(0, \xi)$. Upon some simplification, we thus find that

$$
\begin{array}{r}
\int_{0}^{\xi} \int_{0}^{\xi} \frac{\phi(\xi-v)}{\xi-v} \frac{\phi(\xi-\tau)}{\xi-\tau} \mathcal{F}_{\rho, \lambda}^{\sigma}\left[w(\xi-v)^{\rho}\right] \mathcal{F}_{\rho, \lambda}^{\sigma}\left[w(\xi-\tau)^{\rho}\right] \hbar(v) \hbar(\tau) \mathcal{H}(\tau, v) \mathrm{d} \tau \mathrm{d} v \\
=2\left(\left(\mathcal{T}_{\sigma, \rho, \lambda, 0^{+} ; w^{\prime}}^{\phi} \psi_{1} \psi_{2}\right)(\xi)\left(\mathcal{T}_{\sigma, \rho, \lambda, 0^{+} ; w}^{\phi} \hbar\right)(\xi)\right. \\
\left.-\left(\mathcal{T}_{\sigma, \rho, \lambda, 0^{+} ; w^{\prime}}^{\phi} \hbar \psi_{1}\right)(\xi)\left(\mathcal{T}_{\sigma, \rho, \lambda, 0^{+} ; w}^{\phi} \hbar \psi_{2}\right)(\xi)\right) .
\end{array}
$$

In view of the following known result:

$$
\mathcal{H}(\tau, v)=\int_{\tau}^{v} \int_{\tau}^{v} \psi_{1}^{\prime}(u) \psi_{2}{ }^{\prime}(v) \mathrm{d} u \mathrm{~d} v
$$


if we use the Hölder's inequality for double integrals, we have

$$
\begin{aligned}
|\mathcal{H}(\tau, v)| & \leq\left.\left.\left.\left.\left|\int_{\tau}^{v} \int_{\tau}^{v}\right| \psi_{1}{ }^{\prime}(u)\right|^{r} d u d v\right|^{1 / r}\left|\int_{\tau}^{v} \int_{\tau}^{v}\right| \psi_{2}{ }^{\prime}(u)\right|^{s} \mathrm{~d} u \mathrm{~d} v\right|^{1 / s} \\
& =\left.\left.\left.\left.|\tau-v|\left|\int_{\tau}^{v}\right| \psi_{1}{ }^{\prime}(u)\right|^{r} d u\right|^{1 / r}\left|\int_{\tau}^{v}\right| \psi_{2}{ }^{\prime}(v)\right|^{s} \mathrm{~d} v\right|^{1 / s} .
\end{aligned}
$$

By using (29) in (28), we can deduce that

$$
\begin{aligned}
\left|\int_{0}^{\tau} \int_{0}^{\tau} \frac{\phi(\xi-v)}{\xi-v} \frac{\phi(\xi-\tau)}{\xi-\tau} \mathcal{F}_{\rho, \lambda}^{\sigma}\left[w(\xi-v)^{\rho}\right] \mathcal{F}_{\rho, \lambda}^{\sigma}\left[w(\xi-\tau)^{\rho}\right] \hbar(v) \hbar(\tau) \mathcal{H}(\tau, v) \mathrm{d} \tau \mathrm{d} v\right| \\
\leq \int_{0}^{\xi} \int_{0}^{\tau} \frac{\phi(\xi-v)}{\xi-v} \frac{\phi(\xi-\tau)}{\xi-\tau} \mathcal{F}_{\rho, \lambda}^{\sigma}\left[w(\xi-v)^{\rho}\right] \mathcal{F}_{\rho, \lambda}^{\sigma}\left[w(\xi-\tau)^{\rho}\right] \hbar(v) \hbar(\tau)|\mathcal{H}(\tau, v)| \mathrm{d} \tau \mathrm{d} v \\
\leq \int_{0}^{\xi} \int_{0}^{\tau} \frac{\phi(\xi-v)}{\xi-v} \frac{\phi(\xi-\tau)}{\xi-\tau} \mathcal{F}_{\rho, \lambda}^{\sigma}\left[w(\xi-v)^{\rho}\right] \mathcal{F}_{\rho, \lambda}^{\sigma}\left[w(\xi-\tau)^{\rho}\right] \\
\quad \times\left.\left.\left.\left.|\tau-v| \hbar(v) \hbar(\tau)\left|\int_{\tau}^{v}\right| \psi_{1}{ }^{\prime}(u)\right|^{r} \mathrm{~d} u\right|^{1 / r}\left|\int_{\tau}^{v}\right| \psi_{2}{ }^{\prime}(v)\right|^{s} d v\right|^{1 / s} \mathrm{~d} \tau \mathrm{d} v .
\end{aligned}
$$

By applying the Hölder's inequality to the right-hand side of (30), we get

$$
\begin{aligned}
& \int_{0}^{\xi} \int_{0}^{\xi} \frac{\phi(\xi-v)}{\xi-v} \frac{\phi(\xi-\tau)}{\xi-\tau} \mathcal{F}_{\rho, \lambda}^{\sigma}\left[w(\xi-v)^{\rho}\right] \mathcal{F}_{\rho, \lambda}^{\sigma}\left[w(\xi-\tau)^{\rho}\right] \hbar(v) \hbar(\tau)|\mathcal{H}(\tau, v)| \mathrm{d} \tau \mathrm{d} v \\
& \leq \quad\left(\int_{0}^{\xi} \int_{0}^{\xi} \frac{\phi(\xi-v)}{\xi-v} \frac{\phi(\xi-\tau)}{\xi-\tau} \mathcal{F}_{\rho, \lambda}^{\sigma}\left[w(\xi-v)^{\rho}\right] \mathcal{F}_{\rho, \lambda}^{\sigma}\left[w(\xi-\tau)^{\rho}\right]\right. \\
& \left.\quad \times\left.|\tau-v| \hbar(v) \hbar(\tau)\left|\int_{\tau}^{v}\right| \psi_{1}^{\prime}(u)\right|^{r} \mathrm{~d} u \mid d \tau d v\right)^{1 / r} \\
& \quad \times\left(\int_{0}^{\xi} \int_{0}^{\xi} \frac{\phi(\xi-v)}{\xi-v} \frac{\phi(\xi-\tau)}{\xi-\tau} \mathcal{F}_{\rho, \lambda}^{\sigma}\left[w(\xi-v)^{\rho}\right] \mathcal{F}_{\rho, \lambda}^{\sigma}\left[w(\xi-\tau)^{\rho}\right]\right. \\
& \left.\quad \times\left.|\tau-v| \hbar(v) \hbar(\tau)\left|\int_{\tau}^{v}\right| \psi_{2}^{\prime}(v)\right|^{s} \mathrm{~d} v \mid \mathrm{d} \tau \mathrm{d} v\right)^{1 / s}
\end{aligned}
$$

which, by using the fact that $\psi_{1}{ }^{\prime} \in \mathcal{L}_{r}[0, \infty)$ and $\psi_{2}{ }^{\prime} \in \mathcal{L}_{s}[0, \infty)$, yields

$$
\begin{array}{r}
\int_{0}^{\xi} \int_{0}^{\xi} \frac{\phi(\xi-v)}{\xi-v} \frac{\phi(\xi-\tau)}{\xi-\tau} \mathcal{F}_{\rho, \lambda}^{\sigma}\left[w(\xi-v)^{\rho}\right] \mathcal{F}_{\rho, \lambda}^{\sigma}\left[w(\xi-\tau)^{\rho}\right] \hbar(v) \hbar(\tau)|\mathcal{H}(\tau, v)| \mathrm{d} \tau \mathrm{d} v \\
\leq\left(\left\|\psi_{1}^{\prime}\right\|_{r}^{r} \int_{0}^{\xi} \int_{0}^{\xi} \frac{\phi(\xi-v)}{\xi-v} \frac{\phi(\xi-\tau)}{\xi-\tau} \mathcal{F}_{\rho, \lambda}^{\sigma}\left[w(\xi-v)^{\rho}\right] \mathcal{F}_{\rho, \lambda}^{\sigma}\left[w(\xi-\tau)^{\rho}\right]\right. \\
\times|\tau-v| \hbar(v) \hbar(\tau) \mathrm{d} \tau \mathrm{d} v)^{1 / r} \\
\times\left(\left\|\psi_{2}{ }^{\prime}\right\|_{s}^{s} \int_{0}^{\xi} \int_{0}^{\xi} \frac{\phi(\xi-v)}{\xi-v} \frac{\phi(\xi-\tau)}{\xi-\tau} \mathcal{F}_{\rho, \lambda}^{\sigma}\left[w(\xi-v)^{\rho}\right] \mathcal{F}_{\rho, \lambda}^{\sigma}\left[w(\xi-\tau)^{\rho}\right]\right. \\
\times|\tau-v| \hbar(v) \hbar(\tau) \mathrm{d} \tau \mathrm{d} v)^{1 / s} .
\end{array}
$$


Since $r^{-1}+s^{-1}=1$, it follows that

$$
\begin{aligned}
\int_{0}^{\xi} \int_{0}^{\xi} \frac{\phi(\xi-v)}{\xi-v} \frac{\phi(\xi-\tau)}{\xi-\tau} \mathcal{F}_{\rho, \lambda}^{\sigma}\left[w(\xi-v)^{\rho}\right] \mathcal{F}_{\rho, \lambda}^{\sigma}\left[w(\xi-\tau)^{\rho}\right] \hbar(v) \hbar(\tau)|\mathcal{H}(\tau, v)| \mathrm{d} \tau \mathrm{d} v \\
\leq\left\|\psi_{1}\right\|_{r} \cdot\left\|\psi_{2}{ }^{\prime}\right\|_{s}\left(\int_{0}^{\tau} \int_{0}^{\xi} \frac{\phi(\xi-v)}{\xi-v} \frac{\phi(\xi-\tau)}{\xi-\tau}\right. \\
\left.\quad \times \mathcal{F}_{\rho, \lambda}^{\sigma}\left[w(\xi-v)^{\rho}\right] \mathcal{F}_{\rho, \lambda}^{\sigma}\left[w(\xi-\tau)^{\rho}\right]|\tau-v| \hbar(v) \hbar(\tau) \mathrm{d} \tau \mathrm{d} v\right) .
\end{aligned}
$$

Therefore, by using (30) and (33), we can obtain the first inequality in (25). On the other hand, by using the fact that $0<|\tau-v|<\xi$, we can write

$$
\begin{aligned}
\int_{0}^{\xi} \int_{0}^{\xi} \frac{\phi(\xi-v)}{\xi-v} \frac{\phi(\xi-\tau)}{\xi-\tau} \mathcal{F}_{\rho, \lambda}^{\sigma}\left[w(\xi-v)^{\rho}\right] \mathcal{F}_{\rho, \lambda}^{\sigma}\left[w(\xi-\tau)^{\rho}\right] \hbar(v) \hbar(\tau)|\mathcal{H}(\tau, v)| \mathrm{d} \tau \mathrm{d} v \\
\leq\left\|\psi_{1}{ }^{\prime}\right\|_{r} \cdot\left\|\psi_{2}{ }^{\prime}\right\|_{s} \cdot \xi\left(\int_{0}^{\xi} \int_{0}^{\xi} \frac{\phi(\xi-v)}{\xi-v} \frac{\phi(\xi-\tau)}{\xi-\tau}\right. \\
\left.\quad \times \mathcal{F}_{\rho, \lambda}^{\sigma}\left[w(\xi-v)^{\rho}\right] \mathcal{F}_{\rho, \lambda}^{\sigma}\left[w(\xi-\tau)^{\rho}\right] \hbar(v) \hbar(\tau) \mathrm{d} \tau \mathrm{d} v\right) \\
=\left\|\psi_{1}{ }^{\prime}\right\|_{r} \cdot\left\|\psi_{2}{ }^{\prime}\right\|_{s} \cdot \xi\left[\left(\mathcal{T}_{\sigma, \rho, \lambda, 0^{+} ; w^{\prime}}^{\phi} \hbar\right)(\xi)\right]^{2},
\end{aligned}
$$

which gives the second inequality in (25). The proof of Theorem 4 is thus completed.

Corollary 4. Let $\lambda, \rho>0$ and $w \in \mathbb{R}$. Also let the functions $\psi_{1}$ and $\psi_{2}$ be differentiable on $[0, \infty)$. If

$$
\psi_{1}^{\prime} \in \mathcal{L}_{r}[0, \infty) \quad \text { and } \quad \psi_{2}^{\prime} \in \mathcal{L}_{s}[0, \infty)
$$

with $r>1$ and $r^{-1}+s^{-1}=1$, then

$$
\begin{aligned}
2 \mid & \left(\mathcal{T}_{\sigma, \rho, \lambda, 0^{+} ; w}^{\phi} \psi_{1} \psi_{2}\right)(\xi)\left(\mathcal{T}_{\sigma, \rho, \lambda, 0^{+} ; w^{\prime}}^{\phi} 1\right)(\xi)-\left(\mathcal{T}_{\sigma, \rho, \lambda, 0^{+} ; w}^{\phi} \psi_{1}\right)(\xi)\left(\mathcal{T}_{\sigma, \rho, \lambda, 0^{+} ; w}^{\phi} \psi_{2}\right)(\xi) \mid \\
& \leq\left\|\psi_{1}{ }^{\prime}\right\|_{r} \cdot\left\|\psi_{2}{ }^{\prime}\right\|_{s} \cdot \xi \int_{0}^{\xi} \int_{0}^{\xi} \frac{\phi(\xi-v)}{\xi-v} \frac{\phi(\xi-\tau)}{\xi-\tau} \mathcal{F}_{\rho, \lambda}^{\sigma}\left[w(\xi-v)^{\rho}\right] \mathcal{F}_{\rho, \lambda}^{\sigma}\left[w(\xi-\tau)^{\rho}\right] \mathrm{d} \tau \mathrm{d} v \\
& \leq\left\|\psi_{1}{ }^{\prime}\right\|_{r} \cdot\left\|\psi_{2}{ }^{\prime}\right\|_{s} \cdot \xi\left[\left(\mathcal{T}_{\sigma, \rho, \lambda, 0^{+} ; w^{1}}^{\phi}\right)(\xi)\right]^{2} .
\end{aligned}
$$

Proof. The proof of Corollary 4 follows by applying Theorem 4 for $\hbar \equiv 1$.

Remark 7. From Remark 3, we can derive many other interesting inequalities using our above results. We omit here their proofs and the details are left to the interested reader.

\section{Further Results}

In this last section, we will establish two interesting and useful results in the framework of the defined class of generalized fractional integral operators with respect to another convex function $\Phi$. Some special cases will be discuss in details. Finally, two basic examples will demonstrate the significance of this new results. 
Theorem 5. Let $\lambda, \rho>0$ and $w \in \mathbb{R}$. Also let $\psi$ and $\hbar$ be two positive continuous functions on $\left[\xi_{1}, \infty\right)$, and $\psi \leq \hbar$ on $\left[\xi_{1}, \infty\right)$ for all $\xi_{1} \geq 0$. If $\frac{\psi}{\hbar}$ is decreasing and $\psi$ is increasing on $\left[\xi_{1}, \infty\right)$. Then, for a convex function $\Phi$ with $\Phi(0)=0$, it is asserted that

$$
\frac{\left(\mathcal{T}_{\sigma, \rho, \lambda, \xi_{1}^{+} ; w}^{\phi} \psi\right)(\xi)}{\left(\mathcal{T}_{\sigma, \rho, \lambda, \xi_{1}^{+} ; w^{\prime}}^{\phi}\right)(\xi)} \geq \frac{\left(\mathcal{T}_{\sigma, \rho, \lambda, \xi_{1}^{+} ; w}^{\phi} \Phi(\psi)\right)(\xi)}{\left(\mathcal{T}_{\sigma, \rho, \lambda, \xi_{1}^{+} ; w}^{\phi} \Phi(\hbar)\right)(\xi)} \quad\left(\forall \xi>\xi_{1} \geq 0\right) .
$$

Proof. Since $\Phi$ is convex with $\Phi(0)=0$, the function $\frac{\Phi(\psi(\tau))}{\tau}$ is increasing. As the function $\psi$ is increasing, so is the function $\frac{\Phi(\psi(\tau))}{\psi(\tau)}$. Obviously, the function $\frac{\psi}{\hbar}$ is decreasing. Thus, for all $\tau, v \in\left[\xi_{1}, \infty\right)$, we have

$$
\left(\frac{\Phi(\psi(\tau))}{\psi(\tau)}-\frac{\Phi(\psi(v))}{\psi(v)}\right)\left(\frac{\psi(v)}{\hbar(v)}-\frac{\psi(\tau)}{\hbar(\tau)}\right) \geq 0 .
$$

From (37), it follows that

$$
\frac{\Phi(\psi(\tau))}{\psi(\tau)} \frac{\psi(v)}{\hbar(v)}+\frac{\Phi(\psi(v))}{\psi(v)} \frac{\psi(\tau)}{\hbar(\tau)}-\frac{\Phi(\psi(v))}{\psi(v)} \frac{\psi(v)}{\hbar(v)}-\frac{\Phi(\psi(\tau))}{\psi(\tau)} \frac{\psi(\tau)}{\hbar(\tau)} \geq 0 .
$$

Multiplying (38) by $\hbar(\tau) \hbar(v)$, we get

$$
\begin{aligned}
\frac{\Phi(\psi(\tau))}{\psi(\tau)} \psi(v) \hbar(\tau)+\frac{\Phi(\psi(v))}{\psi(v)} \psi(\tau) \hbar(v)-\frac{\Phi(\psi(v))}{\psi(v)} \psi(v) \hbar(\tau) & \\
& -\frac{\Phi(\psi(\tau))}{\psi(\tau)} \psi(\tau) \hbar(v) \geq 0 .
\end{aligned}
$$

Multiplying (38) by

$$
\frac{\phi(\xi-\tau)}{\xi-\tau} \mathcal{F}_{\rho, \lambda}^{\sigma}\left[w(\xi-\tau)^{\rho}\right]
$$

for all $\tau \in\left(\xi_{1}, \xi\right), \xi>\xi_{1}$ and integrating the result from $\xi_{1}$ to $\xi$, we obtain

$$
\begin{aligned}
& \int_{\xi_{1}}^{\tau} \frac{\phi(\xi-\tau)}{\xi-\tau} \mathcal{F}_{\rho, \lambda}^{\sigma}\left[w(\xi-\tau)^{\rho}\right] \frac{\Phi(\psi(\tau))}{\psi(\tau)} \psi(v) \hbar(\tau) \mathrm{d} \tau \\
& \quad+\int_{\xi_{1}}^{\xi} \frac{\phi(\xi-\tau)}{\xi-\tau} \mathcal{F}_{\rho, \lambda}^{\sigma}\left[w(\xi-\tau)^{\rho}\right] \frac{\Phi(\psi(v))}{\psi(v)} \psi(\tau) \hbar(v) \mathrm{d} \tau \\
& \quad-\int_{\xi_{1}}^{\xi} \frac{\phi(\xi-\tau)}{\xi-\tau} \mathcal{F}_{\rho, \lambda}^{\sigma}\left[w(\xi-\tau)^{\rho}\right] \frac{\Phi(\psi(v))}{\psi(v)} \psi(v) \hbar(\tau) \mathrm{d} \tau \\
& \quad-\int_{\xi_{1}}^{\xi} \frac{\phi(\xi-\tau)}{\xi-\tau} \mathcal{F}_{\rho, \lambda}^{\sigma}\left[w(\xi-\tau)^{\rho}\right] \frac{\Phi(\psi(\tau))}{\psi(\tau)} \psi(\tau) \hbar(v) \mathrm{d} \tau \geq 0 .
\end{aligned}
$$

From (39), we have

$$
\begin{gathered}
\psi(v)\left(\mathcal{T}_{\sigma, \rho, \lambda, \xi_{1}^{+} ; w}^{\phi} \frac{\Phi(\psi)}{\psi} \hbar\right)(\xi)+\left(\frac{\Phi(\psi(v))}{\psi(v)} \hbar(v)\right)\left(\mathcal{T}_{\sigma, \rho, \lambda, \xi_{1}^{+} ; w}^{\phi} \psi\right)(\xi) \\
-\left(\frac{\Phi(\psi(v))}{\psi(v)} \psi(v)\right)\left(\mathcal{T}_{\sigma, \rho, \lambda, \xi_{1}^{+} ; w^{\phi}} \hbar\right)(\xi)-\hbar(v)\left(\mathcal{T}_{\sigma, \rho, \lambda, \xi_{1}^{+} ; w}^{\phi} \frac{\Phi(\psi)}{\psi} \psi\right)(\xi) \geq 0 .
\end{gathered}
$$

Again, multiplying both sides of (40) by

$$
\frac{\phi(\xi-v)}{\xi-v} \mathcal{F}_{\rho, \lambda}^{\sigma}\left[w(\xi-v)^{\rho}\right]
$$


for all $v \in\left(\xi_{1}, \xi\right), \xi>\xi_{1}$ and integrating the result from $\xi_{1}$ to $\xi$, we get

$$
\begin{aligned}
&\left(\mathcal{T}_{\sigma, \rho, \lambda, \xi_{1}^{+} ; w}^{\phi} \psi\right)(\xi)\left(\mathcal{T}_{\sigma, \rho, \lambda, \xi_{1}^{+} ; w}^{\phi} \frac{\Phi(\psi)}{\psi} \hbar\right)(\xi)+\left(\mathcal{T}_{\sigma, \rho, \lambda, \xi_{1}^{+} ; w}^{\phi} \frac{\Phi(\psi)}{\psi} \hbar\right)(\xi) \\
&\left(\mathcal{T}_{\sigma, \rho, \lambda, \xi_{1}^{+} ; w}^{\phi} \psi\right)(\xi) \\
& \geq\left(\mathcal{T}_{\sigma, \rho, \lambda, \xi_{1}^{+} ; w^{+}}^{\phi}\right)(\xi)\left(\mathcal{T}_{\sigma, \rho, \lambda, \xi_{1}^{+} ; w}^{\phi} \Phi(\psi)\right)(\xi) \\
&+\left(\mathcal{T}_{\sigma, \rho, \lambda, \xi_{1}^{+} ; w}^{\phi} \Phi(\psi)\right)(\xi)\left(\mathcal{T}_{\sigma, \rho, \lambda, \xi_{1}^{+} ; w}^{\phi} \hbar\right)(\xi) .
\end{aligned}
$$

From (41), we obtain

$$
\frac{\left(\mathcal{T}_{\sigma, \rho, \lambda, \xi_{1}^{+} ; w}^{\phi} \psi\right)(\xi)}{\left(\mathcal{T}_{\sigma, \rho, \lambda, \xi_{1}^{+} ; w}^{\phi} \hbar\right)(\xi)} \geq \frac{\left(\mathcal{T}_{\sigma, \rho, \lambda, \xi_{1}^{+} ; w}^{\phi} \Phi(\psi)\right)(\xi)}{\left(\mathcal{T}_{\sigma, \rho, \lambda, \xi_{1}^{+} ; w}^{\phi} \frac{\Phi(\psi)}{\psi} \hbar\right)(\xi)} \quad\left(\forall \xi>\xi_{1} \geq 0\right) .
$$

Now, since $\psi \leq \hbar$ on $\left[\xi_{1}, \infty\right)$ for all $\xi_{1} \geq 0$ and $\frac{\Phi(\xi)}{\xi}$ is an increasing function, then for all $\tau \in\left[\xi_{1}, \xi\right)$, we have

$$
\frac{\Phi(\psi(\tau))}{\psi(\tau)} \leq \frac{\Phi(\hbar(\tau))}{\hbar(\tau)}
$$

Multiplying (43)) by

$$
\frac{\phi(\xi-\tau)}{\xi-\tau} \mathcal{F}_{\rho, \lambda}^{\sigma}\left[w(\xi-\tau)^{\rho}\right] \hbar(\tau)
$$

for all $\tau \in\left(\xi_{1}, \xi\right), \xi>\xi_{1}$ and integrating the result from $\xi_{1}$ to $\xi$, we get

$$
\left(\mathcal{T}_{\sigma, \rho, \lambda, \xi_{1}^{+} ; w}^{\phi} \frac{\Phi(\psi)}{\psi} \hbar\right)(\xi) \leq\left(\mathcal{T}_{\sigma, \rho, \lambda, \xi_{1}^{+} ; w}^{\phi} \Phi(\hbar)\right)(\xi) \quad\left(\forall \xi>\xi_{1} \geq 0\right) .
$$

Hence, from (42) and (44), we obtain the desired result (36).

Corollary 5. Under the hypotheses of Theorem 5, if we take

$$
\phi(\xi)=\xi\left(\xi_{2}-\xi\right)^{\alpha-1} \quad\left(\forall \xi \in\left[\xi_{1}, \xi_{2}\right] ; \alpha \in(0,1]\right),
$$

then the following inequality for the so-called conformable left-side fractional integral operator holds true:

$$
\frac{\left(\mathcal{C}_{\sigma, \rho, \lambda, \xi_{1}^{+} ; w}^{\alpha} \psi\right)(\xi)}{\left(\mathcal{C}_{\sigma, \rho, \lambda, \xi_{1}^{+} ; w}^{\alpha} \hbar\right)(\xi)} \geq \frac{\left(\mathcal{C}_{\sigma, \rho, \lambda}^{\alpha} \xi_{\xi_{1}^{+} ; w} \Phi(\psi)\right)(\xi)}{\left(\mathcal{C}_{\sigma, \rho, \lambda, \xi_{1}^{+} ; w}^{\alpha} \Phi(\hbar)\right)(\xi)} \quad\left(\forall \xi>\xi_{1} \geq 0\right) .
$$

Corollary 6. Under the hypotheses of Theorem 5, if we choose

$$
\phi(\xi)=\frac{\xi}{\alpha} \exp (-\mathcal{A} \xi)
$$

where

$$
\mathcal{A}=\frac{1-\alpha}{\alpha}
$$


and $\alpha \in(0,1]$ for all $\xi \in\left[\xi_{1}, \xi_{2}\right]$, then the following inequality for the so-called exponential left-side fractional integral operator holds true:

$$
\frac{\left(\mathcal{E}_{\sigma, \rho, \lambda, \xi_{1}^{+} ; w}^{\alpha} \psi\right)(\xi)}{\left(\mathcal{E}_{\sigma, \rho, \lambda, \xi_{1}^{+} ; w}^{\alpha} \hbar\right)(\xi)} \geq \frac{\left(\mathcal{E}_{\sigma, \rho, \lambda, \xi_{1}^{+} ; w}^{\alpha} \Phi(\psi)\right)(\xi)}{\left(\mathcal{E}_{\sigma, \rho, \lambda, \xi_{1}^{+} ; w}^{\alpha} \Phi(\hbar)\right)(\xi)} \quad\left(\forall \xi>\xi_{1} \geq 0\right) .
$$

Theorem 6. Let $\lambda, \rho>0$ and $w \in \mathbb{R}$. Also let $\psi, \zeta$ and $\hbar$ be three positive continuous functions on $\left[\xi_{1}, \infty\right)$, and $\psi \leq \hbar$ on $\left[\xi_{1}, \infty\right)$ for all $\xi_{1} \geq 0$. If $\frac{\psi}{\hbar}$ is decreasing and the functions $\psi$ and $\zeta$ are increasing on $\left[\xi_{1}, \infty\right)$. Then, for a convex function $\Phi$ with $\Phi(0)=0$, it is asserted that

$$
\frac{\left(\mathcal{T}_{\sigma, \rho, \lambda, \xi_{1}^{+} ; w}^{\phi} \psi\right)(\xi)}{\left(\mathcal{T}_{\sigma, \rho, \lambda, \xi_{1}^{+} ; w^{\prime}}^{\phi} \hbar\right)(\xi)} \geq \frac{\left(\mathcal{T}_{\sigma, \rho, \lambda, \xi_{1}^{+} ; w}^{\phi} \Phi(\psi) \cdot \zeta\right)(\xi)}{\left(\mathcal{T}_{\sigma, \rho, \lambda, \xi_{1}^{+} ; w}^{\phi} \Phi(\hbar) \cdot \zeta\right)(\xi)} \quad\left(\forall \xi>\xi_{1} \geq 0\right) .
$$

Proof. Since $\psi \leq \hbar$ on $\left[\xi_{1}, \infty\right)$ for all $\xi_{1} \geq 0$ and $\frac{\Phi(\xi)}{\tilde{\xi}}$ is an increasing function, then for all $\tau \in\left[\xi_{1}, \xi\right)$, we have

$$
\frac{\Phi(\psi(\tau))}{\psi(\tau)} \leq \frac{\Phi(\hbar(\tau))}{\hbar(\tau)}
$$

Multiplying (48) by

$$
\frac{\phi(\xi-\tau)}{\xi-\tau} \mathcal{F}_{\rho, \lambda}^{\sigma}\left[w(\xi-\tau)^{\rho}\right] \hbar(\tau) \zeta(\tau)
$$

for all $\tau \in\left(\xi_{1}, \xi\right), \xi>\xi_{1}$ and integrating the result from $\xi_{1}$ to $\xi$, we get

$$
\left(\mathcal{T}_{\sigma, \rho, \lambda, \xi_{1}^{+} ; w}^{\phi} \frac{\Phi(\psi)}{\psi} \hbar \zeta\right)(\xi) \leq\left(\mathcal{T}_{\sigma, \rho, \lambda, \xi_{1}^{+} ; w}^{\phi} \Phi(\hbar) \cdot \zeta\right)(\xi) \quad\left(\forall \xi>\xi_{1} \geq 0\right) .
$$

Also, since the function $\Phi$ is convex with $\Phi(0)=0$, then the function $\frac{\Phi(\tau)}{\tau}$ is an increasing function. Since $\psi$ is increasing, so is $\frac{\Phi(\psi(\tau))}{\psi(\tau)}$. Clearly, the function $\frac{\psi}{\hbar}$ is decreasing for all $\tau, v \in\left[\xi_{1}, \xi\right), \xi>\xi_{1}$. Thus

$$
\left(\frac{\Phi(\psi(\tau))}{\psi(\tau)} \zeta(\tau)-\frac{\Phi(\psi(v))}{\psi(v)} \zeta(v)\right)(\psi(v) \hbar(\tau)-\psi(\tau) \hbar(v)) \geq 0 .
$$

From (50), it follows that

$$
\begin{gathered}
\frac{\Phi(\psi(\tau))}{\psi(\tau)} \zeta(\tau) \psi(v) \hbar(\tau)+\frac{\Phi(\psi(v))}{\psi(v)} \zeta(v) \psi(\tau) \hbar(v) \\
-\frac{\Phi(\psi(v))}{\psi(v)} \zeta(v) \psi(v) \hbar(\tau)-\frac{\Phi(\psi(\tau))}{\psi(\tau)} \zeta(\tau) \psi(\tau) \hbar(v) \geq 0 .
\end{gathered}
$$

Multiplying (51) by

$$
\frac{\phi(\xi-\tau)}{\xi-\tau} \mathcal{F}_{\rho, \lambda}^{\sigma}\left[w(\xi-\tau)^{\rho}\right] \hbar(\tau) \zeta(\tau)
$$

for all $\tau \in\left(\xi_{1}, \xi\right), \xi>\xi_{1}$ and integrating the result from $\xi_{1}$ to $\xi$, we obtain

$$
\psi(v)\left(\mathcal{T}_{\sigma, \rho, \lambda, \xi_{1}^{+} ; w}^{\phi} \frac{\Phi(\psi)}{\psi} \hbar \zeta\right)(\xi)+\left(\frac{\Phi(\psi(v))}{\psi(v)} \hbar(v) \zeta(v)\right)\left(\mathcal{T}_{\sigma, \rho, \lambda, \xi_{1}^{+} ; w}^{\phi} \psi\right)(\xi)
$$


$-\left(\frac{\Phi(\psi(v))}{\psi(v)} \psi(v) \zeta(v)\right)\left(\mathcal{T}_{\sigma, \rho, \lambda, \xi_{1}^{+} ; w^{+}}^{\phi}\right)(\xi)-\hbar(v)\left(\mathcal{T}_{\sigma, \rho, \lambda, \xi_{1}^{+} ; w}^{\phi} \frac{\Phi(\psi)}{\psi} \cdot \psi \zeta\right)(\xi) \geq 0$.

Again, multiplying (52) by

$$
\frac{\phi(\xi-v)}{\xi-v} \mathcal{F}_{\rho, \lambda}^{\sigma}\left[w(\xi-v)^{\rho}\right]
$$

for all $v \in\left(\xi_{1}, \xi\right), \xi>\xi_{1}$ and integrating the result from $\xi_{1}$ to $\xi$, we have

$$
\begin{aligned}
\left(\mathcal{T}_{\sigma, \rho, \lambda, \xi_{1}^{+} ; w}^{\phi} \psi\right)(\xi)\left(\mathcal{T}_{\sigma, \rho, \lambda, \xi_{1}^{+} ; w}^{\phi} \frac{\Phi(\psi)}{\psi} \hbar \zeta\right)(\xi)+\left(\mathcal{T}_{\sigma, \rho, \lambda, \xi_{1}^{+} ; w}^{\phi} \frac{\Phi(\psi)}{\psi} \hbar \zeta\right)(\xi) \\
\left(\mathcal{T}_{\sigma, \rho, \lambda, \xi_{1}^{+} ; w}^{\phi} \psi\right)(\xi) \\
\geq\left(\mathcal{T}_{\sigma, \rho, \lambda, \xi_{1}^{+} ; w^{+}}^{\phi}\right)(\xi)\left(\mathcal{T}_{\sigma, \rho, \lambda, \xi_{1}^{+} ; w}^{\phi} \Phi(\psi) \cdot \zeta\right)(\xi) \\
+\left(\mathcal{T}_{\sigma, \rho, \lambda, \xi_{1}^{+} ; w}^{\phi} \Phi(\psi) \cdot \zeta\right)(\xi)\left(\mathcal{T}_{\sigma, \rho, \lambda, \xi_{1}^{+} ; w}^{\phi} \hbar\right)(\xi)
\end{aligned}
$$

From (53), we get

$$
\frac{\left(\mathcal{T}_{\sigma, \rho, \lambda, \xi_{1}^{+} ; w}^{\phi} \psi\right)(\xi)}{\left(\mathcal{T}_{\sigma, \rho, \lambda, \xi_{1}^{+} ; w}^{\phi} \hbar\right)(\xi)} \geq \frac{\left(\mathcal{T}_{\sigma, \rho, \lambda, \xi_{1}^{+} ; w}^{\phi} \Phi(\psi) \cdot \zeta\right)(\xi)}{\left(\mathcal{T}_{\sigma, \rho, \lambda, \xi_{1}^{+} ; w}^{\phi} \frac{\Phi(\psi)}{\psi} \hbar \zeta\right)(\xi)} \quad\left(\forall \xi>\xi_{1} \geq 0\right)
$$

Hence, from (49) and (54), we obtain the required result (47).

Corollary 7. Under the hypotheses of Theorem 6, if we take

$$
\phi(\xi)=\xi\left(\xi_{2}-\xi\right)^{\alpha-1} \quad\left(\forall \xi \in\left[\xi_{1}, \xi_{2}\right] ; \alpha \in(0,1]\right),
$$

then the following inequality for the so-called conformable left-side fractional integral operator holds true:

$$
\frac{\left(\mathcal{C}_{\sigma, \rho, \lambda, \xi_{1}^{+} ; w}^{\alpha} \psi\right)(\xi)}{\left(\mathcal{C}_{\sigma, \rho, \lambda, \xi_{1}^{+} ; w^{\alpha}}^{\alpha} \hbar\right)(\xi)} \geq \frac{\left(\mathcal{C}_{\sigma, \rho, \lambda, \xi_{1}^{+} ; w}^{\alpha} \Phi(\psi) \cdot \zeta\right)(\xi)}{\left(\mathcal{C}_{\sigma, \rho, \lambda, \xi_{1}^{+} ; w}^{\alpha} \Phi(\hbar) \cdot \zeta\right)(\xi)} \quad\left(\forall \xi>\xi_{1} \geq 0\right) .
$$

Corollary 8. Under the hypotheses of Theorem 6, if we choose

$$
\phi(\xi)=\frac{\xi}{\alpha} \exp (-\mathcal{A} \xi),
$$

where

$$
\mathcal{A}=\frac{1-\alpha}{\alpha}
$$

and $\alpha \in(0,1]$ for all $\xi \in\left[\xi_{1}, \xi_{2}\right]$, then the following inequality for the so-called exponential left-side fractional integral operator holds true:

$$
\frac{\left(\mathcal{E}_{\sigma, \rho, \lambda, \xi_{1}^{+} ; w}^{\alpha} \psi\right)(\xi)}{\left(\mathcal{E}_{\sigma, \rho, \lambda, \xi_{1}^{+} ; w^{\alpha}}^{\alpha} \hbar\right)(\xi)} \geq \frac{\left(\mathcal{E}_{\sigma, \rho, \lambda, \xi_{1}^{+} ; w}^{\alpha} \Phi(\psi) \cdot \zeta\right)(\xi)}{\left(\mathcal{E}_{\sigma, \rho, \lambda, \xi_{1}^{+} ; w}^{\alpha} \Phi(\hbar) \cdot \zeta\right)(\xi)} \quad\left(\forall \xi>\xi_{1} \geq 0\right) .
$$




\section{Examples}

Example 1. Assume that $\lambda, \rho>0, r>1$ and $w \in \mathbb{R}$, then the following inequality holds:

$$
\frac{\left(\mathcal{T}_{\sigma, \rho, \lambda, \xi_{1}^{+} ; w}^{\phi} \tau^{2}\right)(\xi)}{\left(\mathcal{T}_{\sigma, \rho, \lambda, \xi_{1}^{+} ; w}^{\phi} \exp (\tau)\right)(\xi)} \geq \frac{\left(\mathcal{T}_{\sigma, \rho, \lambda, \xi_{1}^{+} ; w}^{\phi} \tau^{2 r}\right)(\xi)}{\left(\mathcal{T}_{\sigma, \rho, \lambda, \xi_{1}^{+} ; w}^{\phi} \exp (r \tau)\right)(\xi)} \quad\left(\forall \xi>\xi_{1} \geq 2\right) .
$$

Proof. Taking $\psi(\tau)=\tau^{2}, \hbar(\tau)=\exp (\tau)$ and $\Phi(\tau)=\tau^{r}$, and using Theorem 5 , we get the desired result.

Example 2. Assume that $\alpha \geq 1, \lambda, \rho>0, r>1$ and $w \in \mathbb{R}$, then the following inequality holds:

$$
\frac{\left(\mathcal{T}_{\sigma, \rho, \lambda, \xi_{1}^{+} ; w}^{\phi} \tau^{2}\right)(\xi)}{\left(\mathcal{T}_{\sigma, \rho, \lambda, \xi_{1}^{+} ; w}^{\phi} \exp (\tau)\right)(\xi)} \geq \frac{\left(\mathcal{T}_{\sigma, \rho, \lambda, \xi_{1}^{+} ; w}^{\phi} \tau^{2 r} \cdot \ln (\alpha \tau)\right)(\xi)}{\left(\mathcal{T}_{\sigma, \rho, \lambda, \xi_{1}^{+} ; w}^{\phi} \exp (r \tau) \cdot \ln (\alpha \tau)\right)(\xi)} \quad\left(\forall \xi>\xi_{1} \geq 2\right)
$$

Proof. Choosing $\psi(\tau)=\tau^{2}, \hbar(\tau)=\exp (\tau), \zeta(\tau)=\ln (\alpha \tau)$ and $\Phi(\tau)=\tau^{r}$, and applying Theorem 6, we obtain the desired result.

\section{Conclusions}

In this paper, we have introduced a family of generalized left-side and right-side fractional integral operators with the Wright function as the kernel. We have investigated the Chebyshev inequality via this general family of fractional integral operators. Moreover, we have derived new results of this type of integral inequalities for the finite product of functions. In addition, we have established an estimate for the Chebyshev functional by using our general fractional integral operators. From our above results, we have found similar inequalities for some specialized fractional integrals keeping some of the earlier results in view. Furthermore, two important results and some of their interesting consequences for convex functions in the framework of the defined class of generalized fractional integral operators have been obtained. Finally, two basic examples demonstrated the significance of our results. For future research, in the framework of the defined class of generalized fractional integral operators, we will establish new interesting inequalities using Markov and Minkowski inequalities. From the results derived in this investigation, similar inequalities can be deduced for each of the aforementioned simpler RL fractional integrals with other specialized the FW and ML types kernels.

Author Contributions: Conceptualization, H.M.S., A.K., P.O.M. and K.N.; methodology, H.M.S., P.O.M. and K.N.; software, P.O.M. and A.K.; validation, P.O.M. and K.N.; formal analysis, P.O.M. and K.N.; investigation, H.M.S., P.O.M.; resources, P.O.M., A.K.; data curation, P.O.M. and K.N.; writing-original draft preparation, H.M.S., A.K. and P.O.M.; writing—review and editing, H.M.S., K.N.; visualization, K.N.; supervision, H.M.S. and P.O.M. All authors have read and agreed to the final version of the manuscript.

Funding: This research received no external funding.

Institutional Review Board Statement: Not applicable.

Informed Consent Statement: Not applicable.

Data Availability Statement: Not applicable.

Conflicts of Interest: The authors declare no conflict of interest.

\section{Abbreviations}

The following abbreviations are used in our manuscript: 


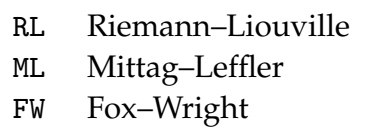

\section{References}

1. Adjabi, Y.; Jarad, F.; Baleanu, D.; Abdeljawad, T. On Cauchy problems with Caputo Hadamard fractional derivatives. Math. Meth. Appl. Sci. 2016, 40, 661-681.

2. Tan, W.; Jiang, F.-L.; Huang, C.-X.; Zhou, L. Synchronization for a class of fractional-order hyperchaotic system and its application. J. Appl. Math. 2012, 2012, 974639. [CrossRef]

3. Zhou, X.-S.; Huang, C.-X.; Hu, H.-J.; Liu, L. Inequality estimates for the boundedness of multilinear singular and fractional integral operators. J. Inequal. Appl. 2013, 2013, 1-15. [CrossRef]

4. Mohammed, P.O.; Machado, J.A.T.; Guirao, J.L.G.; Agarwal, R.P. Adomian decomposition and fractional power series solution of a class of nonlinear fractional differential equations. Mathematics 2021, 9, 1070. [CrossRef]

5. Kilbas, A.A.; Srivastava, H.M.; Trujillo, J.J. Theory and Applications of Fractional Differential Equations; North-Holland Mathematics Studies; Elsevier Science B.V.: Amsterdam, The Netherlands, 2006; Volume 204.

6. Cai, Z.-W.; Huang, J.-H.; Huang, L.-H. Periodic orbit analysis for the delayed Filippov system. Proc. Am. Math. Soc. 2018, 146, 4667-4682. [CrossRef]

7. Chen, T.; Huang, L.-H.; Yu, P.; Huang, W.-T. Bifurcation of limit cycles at infinity in piecewise polynomial systems. Nonlinear Anal. Real World Appl. 2018, 41, 82-106. [CrossRef]

8. Wang, J.-F.; Chen, X.-Y.; Huang, L.-H. The number and stability of limit cycles for planar piecewise linear systems of node-saddle type. J. Math. Anal. Appl. 2019, 469, 405-427. [CrossRef]

9. Aldhaifallah, M.; Tomar, M.; Nisar, K.S.; Purohit, S.D. Some new inequalities for $(k, s)$-fractional integrals. J. Nonlinear Sci. Appl. 2016, 9, 5374-5381. [CrossRef]

10. Houas, M. Certain weighted integral inequalities involving the fractional hypergeometric operators. Scientia Ser. A Math. Sci. 2016, 27, 87-97.

11. Houas, M. On some generalized integral inequalities for Hadamard fractional integrals. Mediterr. J. Model. Simul. 2018, 9, 43-52.

12. Mohammed, P.O.; Abdeljawad, T.; Alqudah, M.A.; Jarad, F. New discrete inequalities of Hermite-Hadamard type for convex functions. Adv. Differ. Equ. 2021, 2021, 122. [CrossRef]

13. Mohammed, P.O.; Sarikaya, M.Z.; Baleanu, D. On the generalized Hermite-Hadamard inequalities via the tempered fractional integrals. Symmetry 2020, 12, 595. [CrossRef]

14. Cloud, M.J.; Drachman, B.C.; Lebedev, L. Inequalities, 2nd ed.; Springer: Cham, Switzerland, 2014.

15. Atıc1, F.M.; Yaldız, H. Convex functions on discrete time domains. Canad. Math. Bull. 2016, 59, 225-233. [CrossRef]

16. Khan, M.B.; Mohammed, P.O.; Noor, M.A.; Hamed, Y.S. New Hermite-Hadamard inequalities in fuzzy-interval fractional calculus and related inequalities. Symmetry 2021, 13, 673. [CrossRef]

17. Khan, M.B.; Mohammed, P.O.; Noor, M.A.; Abualnaja, K.M. Fuzzy integral inequalities on coordinates of convex fuzzy intervalvalued functions. Math. Biosci. Eng. 2021, 18, 6552-6580. [CrossRef]

18. Sana, G.; Mohammed, P.O.; Shin, D.Y.; Noor, M.A.; Oudat, M.S. On iterative methods for solving nonlinear equations in quantum calculus. Fractal Fract. 2021, 5, 60. [CrossRef]

19. Khan, M.B.; Srivastava, H.M.; Mohammed, P.O.; Guirao, J.L.G. Fuzzy mixed variational-like and integral inequalities for strongly preinvex fuzzy mappings. Symmetry 2021, 13, 1816. [CrossRef]

20. Miller, K.S.; Ross, B. An Introduction to the Fractional Calculus and Fractional Differential Equations; Wiley: New York, NY, USA, 1993.

21. Samko, S.G.; Kilbas, A.A.; Marichev, O.I. Fractional Integrals and Derivatives: Theory and Applications; Gordon \& Breach Science Publishers: Yverdon, Switzerland, 1993.

22. Herrmann, R. Fractional Calculus: An Introduction for Physicists; World Scientific: Singapore, 2011.

23. Alsmeyer, G. Chebyshev's Inequality. In International Encyclopedia of Statistical Science; Lovric, M., Ed.; Springer: Berlin/Heidelberg, Germany; New York, NY, USA, 2011.

24. Chebyshev, P.L. Sur les expressions approximatives des integrales definies par les autres prises entre les mêmes limites. Proc. Math. Soc. Charkov 1882, 2, 93-98.

25. Baleanu, D.; Purohit, S.D. Chebyshev type integral inequalities involving the fractional hypergeometric operators. Abstr. Appl. Anal. 2014, 2014, 609160. [CrossRef]

26. Rahman, G.; Ullah, Z.; Khan, A.; Set, E.; Nisar, K.S. Certain Chebyshev-type inequalities involving fractional conformable integral operators. Mathematics 2019, 7, 364. [CrossRef]

27. Ntouyas, S.K.; Purohit, S.D.; Tariboon, J. Certain Chebyshev type integral inequalities involving Hadamard's fractional operators. Abst. Appl. Anal. 2014, 2014, 249091. [CrossRef]

28. Srivastava, H.M.; Kashuri, A.; Mohammed, P.O.; Alsharif, A.M.; Guirao, J.L.G. New Chebyshev type inequalities via a general family of fractional integral operators with a modified Mittag-Leffler kernel. AIMS Math. 2021, 6, 11167-11186. [CrossRef]

29. Rahman, G.; Nisar, K.S.; Abdeljawad, T. Certain new proportional and Hadamard proportional fractional integral inequalities. J. Inequal. Appl. 2021, 71,1-14. 
30. Nisar, K.S.; Rahman, G.; Baleanu, D.; Samraiz, M.; Iqbal, S. On the weighted fractional Pólya-Szegö and Chebyshev-types integral inequalities concerning another function. Adv. Differ. Equ. 2020, 623, 1-18. [CrossRef]

31. Rahman, G.; Abdeljawad, T.; Jarad, F.; Khan, A.; Nisar, K.S. Certain inequalities via generalized proportional Hadamard fractional integral operators. Adv Differ. Equ. 2019, 454, 1-10. [CrossRef]

32. Rahman, G.; Nisar, K.S.; Khan, S.U.; Baleanu, D.; Vijayakumar, V. On the weighted fractional integral inequalities for Chebyshev functionals. Adv Differ. Equ. 2021, 18, 1-19.

33. Rahman, G.; Nisar, K.S.; Abdeljawad, T. Certain Hadamard proportional fractional integral inequalities. Mathematics 2020, 8, 504. [CrossRef]

34. Rahman, G.; Nisar, K.S.; Abdeljawad, T.; Ullah, S. Certain fractional proportional integral inequalities via convex functions Mathematics 2020, 8, 222. [CrossRef]

35. Rahman, G.; Abdeljawad, T.; Jarad, F.; Nisar, K.S. Bounds of generalized proportional fractional integrals in general form via convex functions and their applications. Mathematics 2020, 8, 113. [CrossRef]

36. Dahmani, Z. About some integral inequalities using Riemann-Liouville integrals. Gen. Math. 2012, $20,63-69$.

37. Niculescu, C.P.; Roventa, I. An extention of Chebyshev's algebric inequality. Math. Reports 2013, 15, 91-95.

38. Özdemir, M.E.; Set, E.; Akdemir, A.O.; Sarikaya, M.Z. Some new Chebyshev type inequalities for functions whose derivatives belongs to spaces. Afr. Mat. 2015, 26, 1609-1619. [CrossRef]

39. Usta, F.; Budak, H.; Sarikaya, M.Z. On Chebyshev type inequalities for fractional integral operators. AIP Conf. Proc. 2017, 1833, $1-4$.

40. Usta, F.; Budak, H.; Sarikaya, M.Z. Some new Chebyshev type inequalities utilizing generalized fractional integral operators. AIMS Math. 2020, 5, 1147-1161. [CrossRef]

41. Pachpatte, B.G. A note on Chebyshev-Grüss type inequalities for differential functions. Tamsui Oxford J. Math. Sci. 2006, 22, 29-36.

42. Liu, Z. A variant of Chebyshev inequality with applications. J. Math. Inequal. 2013, 7, 551-561. [CrossRef]

43. Set, E.; Dahmani, Z.; Muncu, İ. New extensions of Chebyshev type inequalities using generalized Katugampola integrals via Pólya-Szegö inequality. Int. J. Optim. Control Theory Appl. (IJOCTA) 2018, 8, 137-144. [CrossRef]

44. Set, E.; Özdemir, M.E.; Demirbaş, S. Chebyshev type inequalities involving extended generalized fractional integral operators. AIMS Math. 2020, 5, 3573-3583. [CrossRef]

45. Baleanu, D.; Fernandez, A. On fractional operators and their classifications. Mathematics 2019, 7, 830. [CrossRef]

46. Hilfer, R.; Luchko, Y.; Desiderata for fractional derivatives and integrals. Mathematics 2019, 7, 149. [CrossRef]

47. Teodoro, G.S.; Machado, J.A.T.; Oliveira, E.C. A review of definitions of fractional derivatives and other operators. J. Comput. Phys. 2019, 388, 195-208. [CrossRef]

48. Atangana, A. Fractional Operators with Constant and Variable Order with Application to Geo-Hydrology; Academic Press: New York, NY, USA, 2017.

49. Hristov, J. (Ed.) The Craft of Fractional Modelling in Science and Engineering; MDPI: Basel, Switzerland, 2018.

50. Ata, E.; Kiymaz, İ.O. A study on certain properties of generalized special functions defined by Fox-Wright function. Appl. Math Nonlinear Sci. 2020, 5, 147-162. [CrossRef]

51. İlhan, E.; Kıymaz, İ.O. A generalization of truncated $M$-fractional derivative and applications to fractional differential equations. Appl. Math. Nonlinear Sci. 2020, 5, 171-188. [CrossRef]

52. Şahin, R.; Yağci, O. Fractional calculus of the extended hypergeometric function. Appl. Math. Nonlinear Sci. 2020, 5, 369-384. [CrossRef]

53. Kaur, D.; Agarwal, P.; Rakshit, M.; Chand, M. Fractional calculus involving $(p, q)$-Mathieu type series. Appl. Math. Nonlinear Sci. 2020, 5, 15-34. [CrossRef]

54. Kabra, S.; Nagar, H.; Nisar, K.S.; Suthar, D.L. The Marichev-Saigo-Maeda fractional calculus operators pertaining to the generalized $k$-Struve function. Appl. Math. Nonlinear Sci. 2020, 5, 593-602. [CrossRef]

55. Mohammed, P.O.; Abdeljawad, T.; Baleanu, D.; Kashuri, A.; Hamasalh, F.; Agarwal, P. New fractional inequalities of HermiteHadamard type involving the incomplete gamma functions. J. Inequal. Appl. 2020, 2020, 263. [CrossRef]

56. Gorenflo, R.; Kilbas, A.A.; Mainardi, F.; Rogosin, S.V. Mittag-Leffler Functions: Related Topics and Applications; Springer: Berlin, Germany, 2014.

57. Gorenflo, R.; Mainardi, F.; Srivastava, H.M. Special functions in fractional relaxation-oscillation and fractional diffusion-wave phenomena. In Proceedings of the Eighth International Colloquium on Differential Equations, Plovdiv, Bulgaria, 18-23 August 1997; Bainov, D., Ed.; VSP Publishers: Utrecht, The Netherlands; Tokyo, Japan, 1998; pp. 195-202.

58. Fernandez, A.; Mohammed, P. Hermite-Hadamard inequalities in fractional calculus defined using Mittag-Leffler kernels. Math. Meth. Appl. Sci. 2020,1-18. [CrossRef]

59. Mohammed, P.O.; Abdeljawad, T. Integral inequalities for a fractional operator of a function with respect to another function with nonsingular kernel. Adv. Differ. Equ. 2020, 2020, 363. [CrossRef]

60. Mathai, A.M.; Haubold, H.J. Mittag-Leffler Functions and Fractional Calculus. In Special Functions for Applied Scientists; Springer: New York, NY, USA, 2008.

61. Srivastava, H.M. Operators of basic (or $q$-) calculus and fractional $q$-calculus and their applications in geometric function theory of complex analysis. Iran. J. Sci. Technol. Trans. A Sci. 2020, 44, 327-344. [CrossRef] 
62. Srivastava, H.M. Some families of Mittag-Leffler type functions and associated operators of fractional calculus. TWMS J. Pure Appl. Math. 2016, 7, 123-145.

63. Srivastava, H.M.; Bansal, M.K.; Harjule, P. A study of fractional integral operators involving a certain generalized multi-index Mittag-Leffler function. Math. Meth. Appl. Sci. 2018, 41, 6108-6121. [CrossRef]

64. Srivastava, H.M.; Bansal, M.K.; Harjule, P. A class of fractional integral operators involving a certain general multi-index Mittag-Leffler function. Ukr. Math. J. 2020, in press.

65. Fox, C. The asymptotic expansion of generalized hypergeometric functions. Proc. Lond. Math. Soc. 1928, 27, 389-400. [CrossRef]

66. Wright, E.M. The asymptotic expansion of the generalized hypergeometric function. J. Lond. Math. Soc. 1935, 10, $286-293$. [CrossRef]

67. Wright, E.M. The asymptotic expansion of the generalized hypergeometric function. Proc. Lond. Math. Soc. 1940, 46, 389-408. [CrossRef]

68. Srivastava, H.M.; Karlsson, P.W. Multiple Gaussian Hypergeometric Series; Halsted Press (Ellis Horwood Limited, Chichester), John Wiley and Sons: New York, NY, USA, 1985.

69. Wright, E.M. The asymptotic expansion of integral functions defined by Taylor series. Philos. Trans. R. Soc. Lond. Ser. A Math. Phys. Sci. 1940, 238, 423-451.

70. Srivastava, H.M.; Kashuri, A.; Mohammed, P.O.; Baleanu, D. Fractional integral inequalities for exponentially nonconvex functions and their applications. Fractal Fract. 2021, 5, 80. [CrossRef]

71. Raina, R.K. On generalized Wright's hypergeometric functions and fractional calculus operators. East Asian Math. J. 2005, 21, 191-203.

72. Srivastava, H.M. Some parametric and argument variations of the operators of fractional calculus and related special functions and integral transformations. J. Nonlinear Convex Anal. 2021, 22, 1501-1520.

73. Agarwal, R.P.; Luo, M.J.; Raina, R.K. On Ostrowski type inequalities. Fasc. Math. 2016, 56, 5-27. [CrossRef]

74. Sarikaya, M.Z.; Ertuğral, F. On the generalized Hermite-Hadamard inequalities. Ann. Univ. Craiova Math. Comput. Sci. Ser. 2020, 47, 193-213. 